\title{
Wind Turbine Generator System Power Performance Test Report for the Gaia-Wind 11-kW Wind Turbine
}

A. Huskey, A. Bowen, and D. Jager

Technical Report NREL/TP-500-46151

December 2009

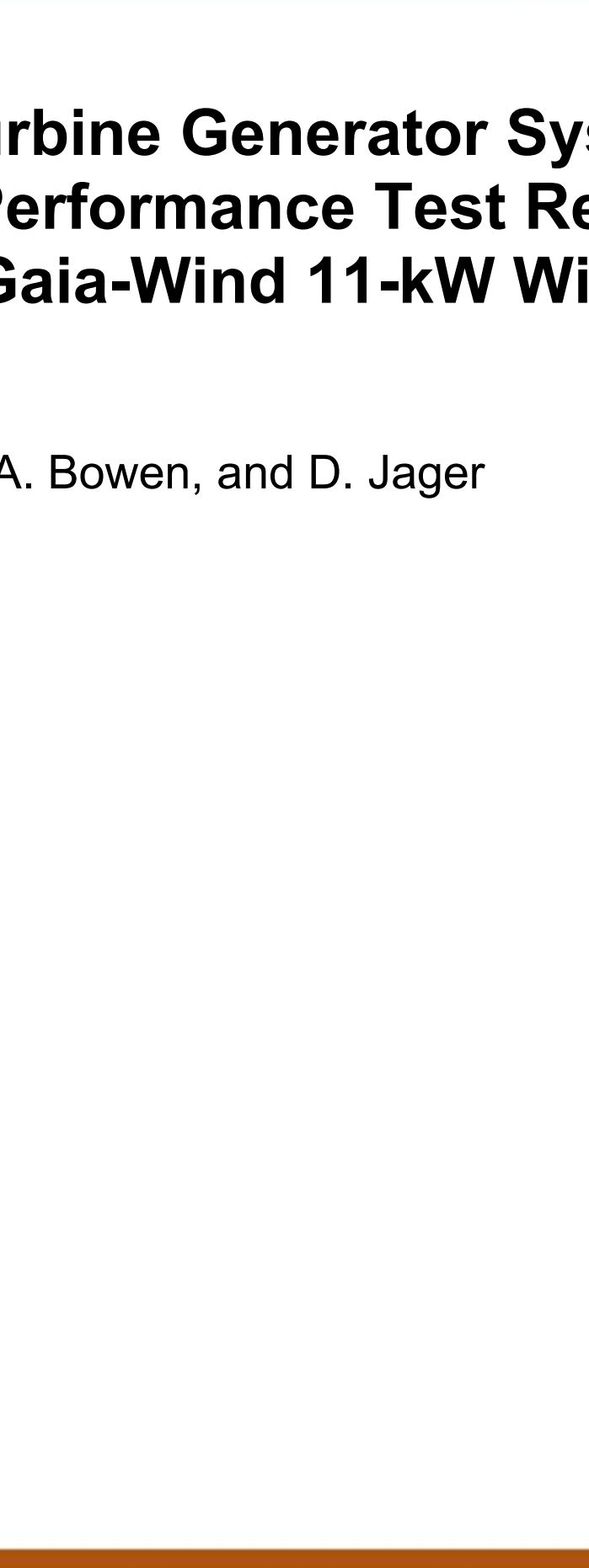




\title{
Wind Turbine Generator System \\ Power Performance Test Report \\ for the
}

\section{Gaia-Wind 11-kW Wind Turbine}

Conducted for

\author{
National Renewable Energy Laboratory \\ 1617 Cole Blvd. \\ Golden, CO 80401 \\ Conducted by \\ National Wind Technology Center \\ National Renewable Energy Laboratory \\ 1617 Cole Blvd. \\ Golden, CO 80401
}

Arlinda Huskey, Amy Bowen, and Dave Jager

June 11, 2009 


\section{Notice}

This report was prepared by the National Renewable Energy Laboratory (NREL), operated for the United States Department of Energy (DOE) by the Alliance for Sustainable Energy, LLC (Alliance), as an account of work sponsored by the United States government. The test results documented in this report define the characteristics of the test article as configured and under the conditions tested.

THIS REPORT IS PROVIDED "AS IS" AND NEITHER THE GOVERNMENT, ALLIANCE, NREL NOR ANY OF THEIR EMPLOYEES, MAKES ANY WARRANTY, EXPRESS OR IMPLIED, INCLUDING THE WARRANTIES OF MERCHANTABILITY AND FITNESS FOR A PARTICULAR PURPOSE, OR ASSUMES ANY LEGAL LIABILITY OR RESPONSIBILITY FOR THE ACCURACY, COMPLETENESS, OR USEFULNESS OF ANY SUCH INFORMATION DISCLOSED IN THE REPORT, OR OF ANY APPARATUS, PRODUCT, OR PROCESS DISCLOSED, OR REPRESENTS THAT ITS USE WOULD NOT INFRINGE PRIVATELY OWNED RIGHTS.

Neither Alliance nor the U. S. Government shall be liable for special, consequential or incidental damages. Reference herein to any specific commercial product, process, or service by trade name, trademark, manufacturer, or otherwise does not necessarily constitute or imply its endorsement, recommendation, or favoring by the United States government or any agency thereof. The views and opinions of the authors expressed herein do not necessarily state or reflect those of the United States government or any agency thereof or Alliance.

NREL is a DOE Laboratory, and as an adjunct of the United States government, cannot certify wind turbines. The information in this report is limited to NREL's knowledge and understanding as of this date.

NREL is accredited by the American Association for Laboratory Accreditation (A2LA) and the results shown in this test report have been determined in accordance with the NREL's terms of accreditation unless stated otherwise in the report.

This report shall not be reproduced, except in full, without the written approval of Alliance or successor operator of NREL.

Approval By:

$$
\text { Arlinda Huskey, NREL Test Engineer Date }
$$

\section{Review By:}




\section{Contents}

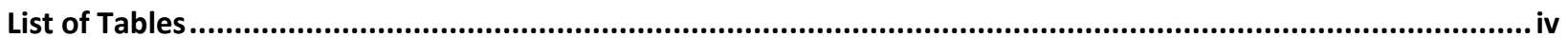

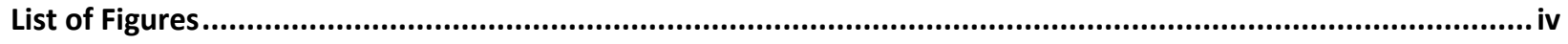

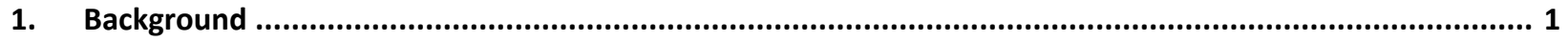

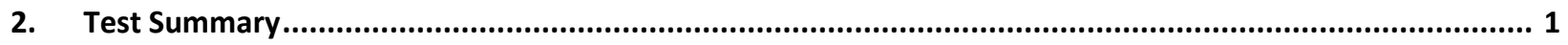

3. Test Turbine Configuration...................................................................................................

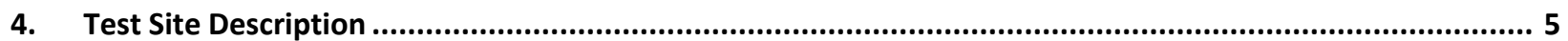

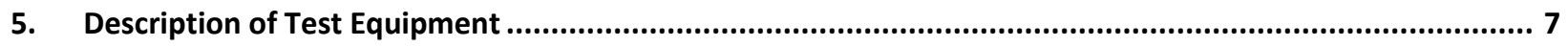

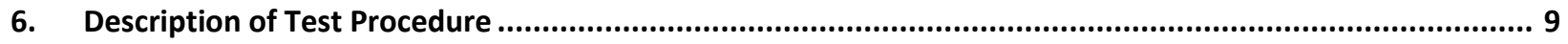

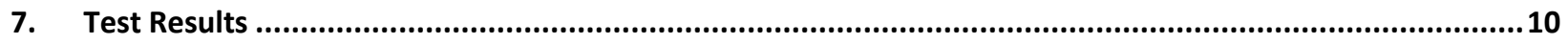

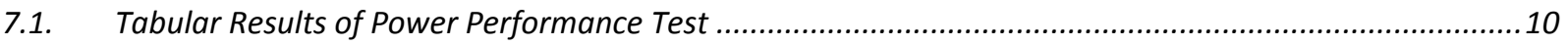

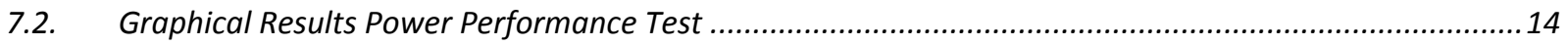

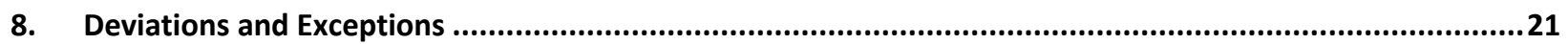

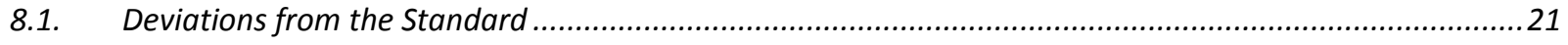

8.2. Exceptions to NWTC-CT Quality Assurance System ......................................................................21

A. Appendix - Photographs of the Test Site from the Turbine Base....................................................22

B. Appendix - Equipment Calibration Sheets...............................................................................28 


\section{List of Tables}

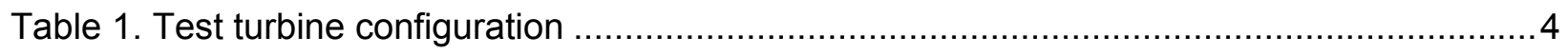

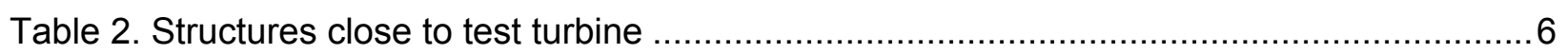

Table 3. Criteria for test site without site calibration ..........................................................

Table 4. Equipment used in the power performance test .................................................

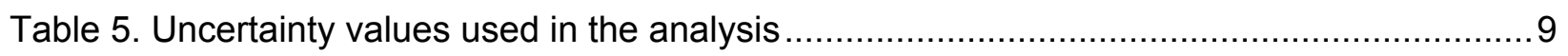

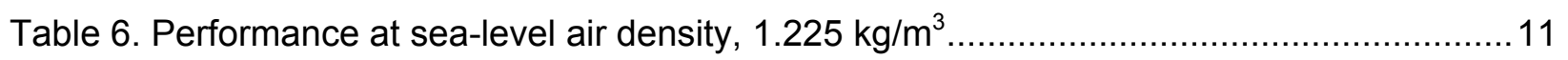

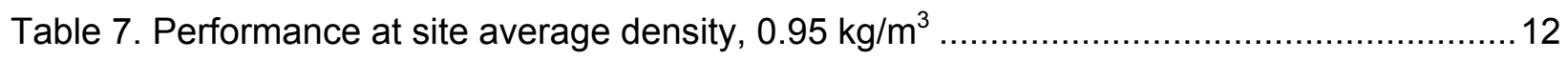

Table 8. Annual energy production at sea-level density, $1.225 \mathrm{~kg} / \mathrm{m}^{3} \ldots \ldots \ldots \ldots \ldots \ldots \ldots \ldots \ldots \ldots \ldots . . .13$

Table 9. Annual energy production at site average density, $0.95 \mathrm{~kg} / \mathrm{m}^{3} \ldots \ldots \ldots \ldots \ldots \ldots \ldots \ldots \ldots \ldots . . .13$

\section{List of Figures}

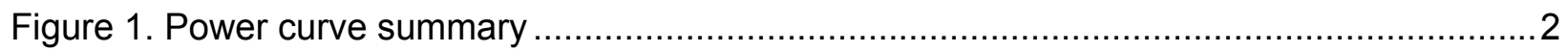

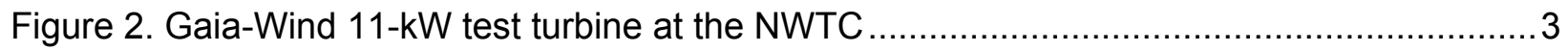

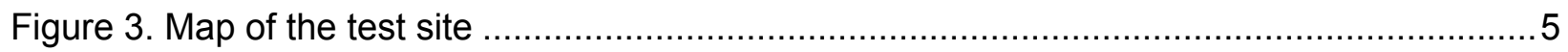

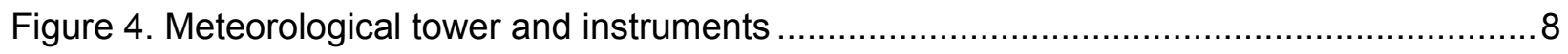

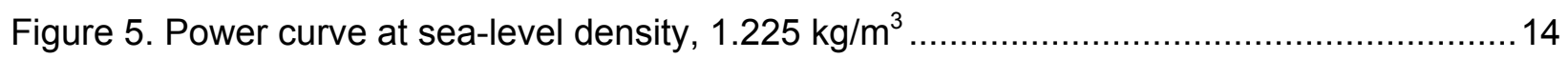

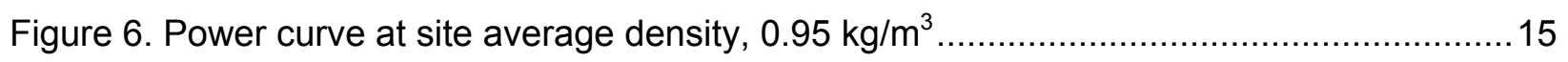

Figure 7. Scatter plot of mean, standard deviation, minimum, and maximum power data .........16

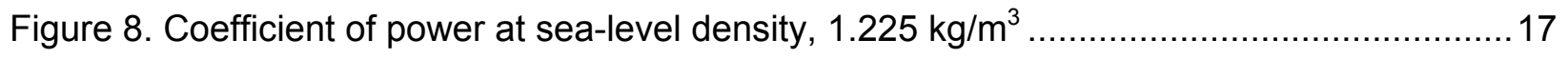

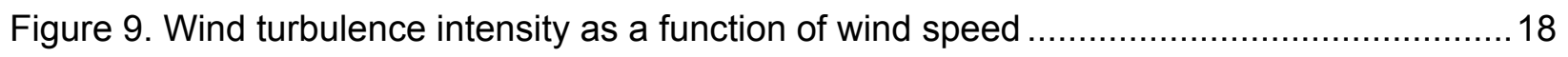

Figure 10. Wind speed and turbulence intensity as a function of wind direction........................19

Figure 11. Rotor speed as a function of wind speed (1-minute averages) and binned values ...20 


\section{Background}

This test is being conducted as part of the U.S. Department of Energy's (DOE) Independent Testing project. This project was established to help reduce the barriers of wind energy expansion by providing independent testing results for small turbines. In total, four turbines are being tested at the National Wind Technology Center (NWTC) as a part of this project. Power performance testing is one of up to 5 tests that may be performed on the turbines, including duration, safety and function, noise, and power quality tests.

\section{Test Summary}

Figure 1 is a summary of the results of a power performance test that the National Renewable Energy Laboratory (NREL) conducted on the Gaia-Wind 11-kW small wind turbine (shown in Figure 2). In this test, the Gaia-Wind 11-kW turbine was installed at the NWTC near Boulder, Colorado. This test was conducted in accordance with the International Electrotechnical Commission (IEC) standard, Wind Turbine Generator Systems Part 12: Power Performance Measurements of Electricity Producing Wind Turbines, IEC 61400-12-1 Ed.1.0, 2005-12. Because the Gaia-Wind 11-kW is a small turbine according to the IEC definition, NREL also followed Annex $\mathrm{H}$, which applies to small wind turbines. This test report refers to these procedures as the "Standard."

In these summary results, power is normalized to sea-level air density. This test began on June 9, 2008, and ended on October 27, 2008. In all, NREL collected 1070.03 hours of valid data during that period. The highest bin filled was the $20.0 \mathrm{~m} / \mathrm{s}$ bin. The amount of test data is sufficient to meet the requirements of the Standard. 
Power Perform ance Test

Gaia-W ind 11-kW

Sea-Level Air Density Nom alized Power Curve

Iurbine Specifications:

Serial Numbe

Rated Power:

Cut-in Wind Speed:

Cut-out Wind Speso:

Rated Wind Speed:

Rotor Diameter:

Contral Type

Pitch Setting:

Site Conditions:

Location:

Aversge Air Dens ity:

Meas urement Sectors:

NW TC, Boulder, CO

$0.97 \quad \mathrm{~kg} / \mathrm{m}^{3}$

257-332 degrees true

Teq Statistics

Start Dete

End Date:

Amount of Data Collected:

Highest Bin Filled

Test Completed?
10711114

$\begin{array}{rr}11 & \mathrm{~kW} \\ 3.50 & \mathrm{~m} / \mathrm{s} \\ 25 & \mathrm{~m} / \mathrm{s} \\ 9.5 & \mathrm{~m} / \mathrm{s} \\ 13 & \mathrm{~m}\end{array}$

Stall

Fixed

\begin{tabular}{|c|c|c|c|}
\hline $\begin{array}{c}\text { Bin Wind } \\
\text { Speed } \\
\text { (m/s) }\end{array}$ & $\begin{array}{l}\text { Bin } \\
\text { Power } \\
\text { (kWV) }\end{array}$ & $\begin{array}{l}\text { Number } \\
\text { Data } \\
\text { Points }\end{array}$ & $C_{p}$ \\
\hline 0.49 & -0.08 & 1,313 & -8.43 \\
\hline 1.03 & -0.08 & 2,467 & -0.93 \\
\hline 1.52 & -0.08 & 4,301 & -0.29 \\
\hline 201 & 0.08 & 6.209 & -0.12 \\
\hline 2.50 & -0.05 & 7,672 & -0.07 \\
\hline 300 & 0.10 & 7,347 & -0.04 \\
\hline 3.49 & 0.11 & 6.270 & -0.03 \\
\hline 3.99 & 0.31 & 5,193 & 0.06 \\
\hline 4.49 & t. 15 & 3.951 & 0.16 \\
\hline 499 & 228 & 3,021 & 0.23 \\
\hline 5.49 & 3.67 & 2.351 & 0.27 \\
\hline 599 & 5.00 & 2.017 & 029 \\
\hline 6.49 & 6.27 & 1,706 & 0.28 \\
\hline 700 & 7.57 & 1,455 & 027 \\
\hline 7.49 & 8.70 & 1,358 & 0.25 \\
\hline 799 & 9.80 & 1,142 & 024 \\
\hline 8.49 & 10.77 & 970 & 0.22 \\
\hline 9.00 & 11.67 & 873 & 020 \\
\hline 9.50 & 12.36 & 713 & 0.18 \\
\hline 10.00 & 13.12 & 604 & 0.16 \\
\hline 10.49 & 13.69 & 520 & 0.15 \\
\hline 11.00 & 14.15 & 417 & 0.13 \\
\hline 11.49 & 1459 & 336 & 0.12 \\
\hline 12.00 & 1430 & 317 & 0.11 \\
\hline 12.49 & 14.90 & 280 & 000 \\
\hline 13.00 & 15.02 & 225 & 008 \\
\hline 13.49 & 1493 & 137 & 007 \\
\hline 13.99 & 1430 & 180 & 0.07 \\
\hline 14.48 & 14.60 & 146 & 0.06 \\
\hline 15.00 & 14.40 & 117 & 005 \\
\hline 15.48 & 14.49 & 100 & 005 \\
\hline 16.03 & 14.42 & 37 & 004 \\
\hline 16.50 & 14.15 & 70 & 004 \\
\hline 1699 & 14.24 & 66 & 004 \\
\hline 17.50 & 1413 & 59 & 003 \\
\hline 1799 & 1403 & 53 & 0.03 \\
\hline 18.43 & 13.91 & 31 & 003 \\
\hline 18.99 & 1400 & 25 & 0.03 \\
\hline 19.45 & 14.11 & 13 & 002 \\
\hline 19.92 & 13.74 & 10 & 002 \\
\hline
\end{tabular}

Sea-Level Air Density Normalized Power Curve

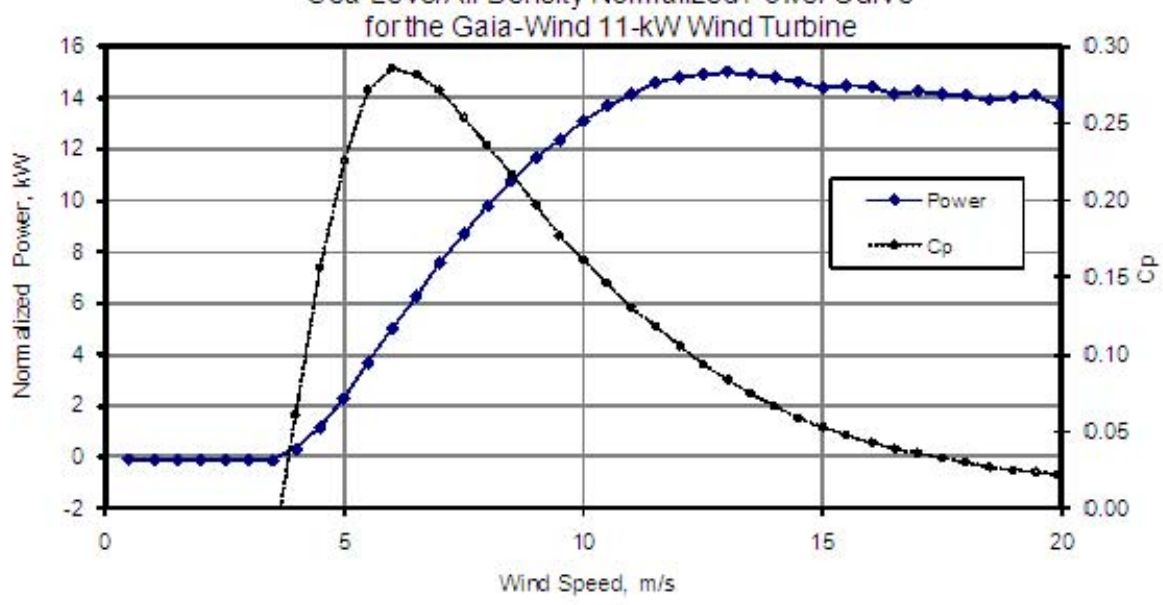

Figure 1. Power curve summary 


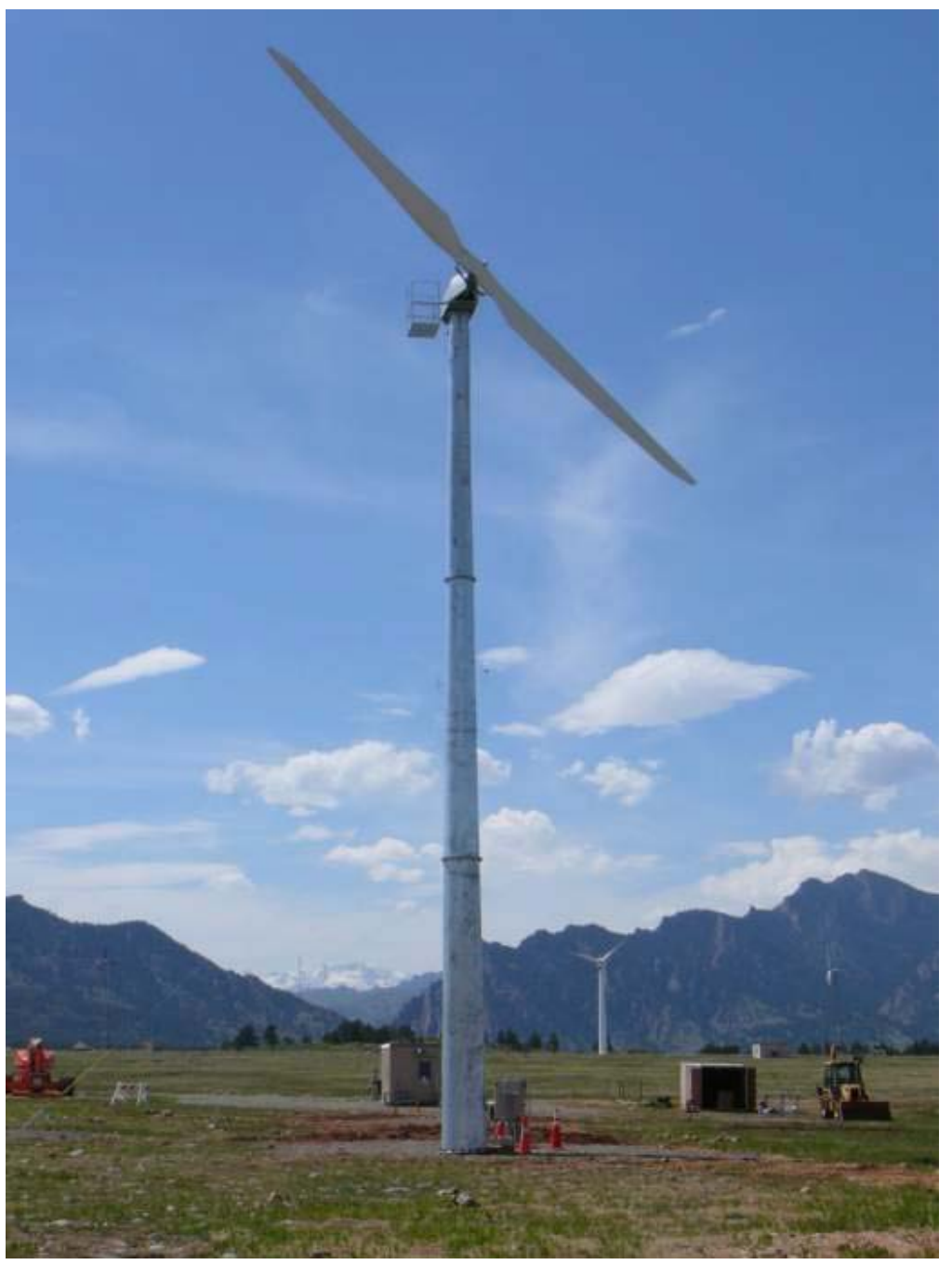

Figure 2. Gaia-Wind 11-kW test turbine at the NWTC 


\section{Test Turbine Configuration}

Table 1 lists the configuration of the Gaia-Wind 11-kW that was tested at the NWTC.

Table 1. Test turbine configuration

\begin{tabular}{|l|l|}
\hline $\begin{array}{l}\text { Turbine make, model, serial number, production } \\
\text { year }\end{array}$ & Gaia-Wind 11-kW, 10711114, 2007 \\
\hline Rotor diameter $(\mathrm{m})$ & 13 \\
\hline Hub height $(\mathrm{m})$ & 18.2 \\
\hline Tower type & Tubular \\
\hline Rated electrical power $(\mathrm{kW})$ & 11 \\
\hline Rated wind speed $(\mathrm{m} / \mathrm{s})$ & 9.5 \\
\hline Rotor speed range $(\mathrm{rpm})$ & $56-62$ \\
\hline Fixed or variable pitch & Fixed blade, variable tip \\
\hline Number of blades & 2 \\
\hline Blade tip pitch angle (deg) & 90 \\
\hline Blade make, type, serial number & Gaia-Wind T202, glass fiber, centrifugally \\
& activated tip brake, 2007/22 \\
\hline Control system (device and software version) & Gaia-Wind IC-1000, Rev.1:P00515।031020 \\
\hline
\end{tabular}

Measurements verified the rotor diameter. The tip pitch changed when deployed. 


\section{Test Site Description}

The test turbine is located at site 3.3B at the NWTC, which is 8 miles south of Boulder, Colorado. The terrain consists of mostly flat terrain with short vegetation (see Appendix A for photos of the test site). The site has prevailing winds bearing 292 degrees relative to true north. For measurements for which it is important to accurately measure wind speed, NREL uses data obtained when the wind direction is between 257 and 332, and between 147 and 197 degrees true. In this measurement sector, the influence of terrain and obstructions on the anemometer is small. Figure 3 shows the turbine and meteorological tower locations as well as nearby obstructions and topographical features of the site. Table 2 gives sizes and distances of nearby obstructions.

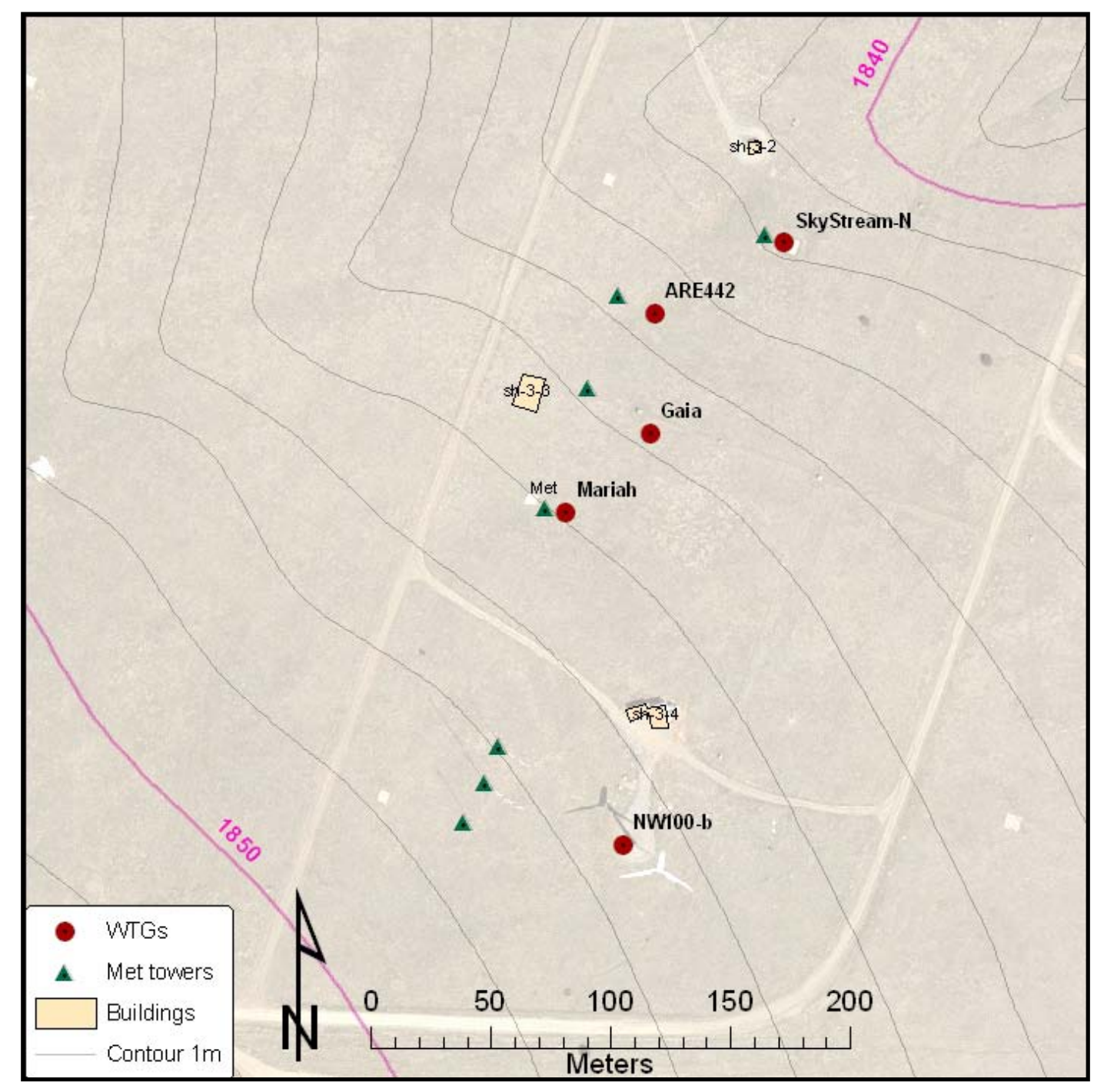

Figure 3. Map of the test site 
Table 2. Structures close to test turbine

\begin{tabular}{|l|c|c|c|c|}
\hline Designation & $\begin{array}{c}\text { Bearing } \\
\text { from } \\
\text { Test } \\
\text { Turbine } \\
\text { (degrees T) }\end{array}$ & $\begin{array}{c}\text { Distance from } \\
\text { Test Turbine } \\
(\mathrm{m})\end{array}$ & $\begin{array}{c}\text { Obstruction } \\
\text { Height } \\
(\mathrm{m})\end{array}$ & $\begin{array}{c}\text { Rotor } \\
\text { Diameter or } \\
\text { Obstruction } \\
\text { Width } \\
(\mathrm{m})\end{array}$ \\
\hline Met B & 290 & 107.5 & 18.2 & 0.4 \\
\hline Data shed & 292 & 207.5 & 3.1 & 7.0 \\
\hline ARE & 2 & 157.6 & 31.0 & 7.3 \\
\hline Met A & 344 & 184.9 & 31.0 & 0.4 \\
\hline Mariah & 227 & 168.9 & 6.1 & 1.2 \\
\hline Met C & 236 & 184.5 & 4.6 & 0.4 \\
\hline
\end{tabular}

NREL completed a site assessment to determine if the site fails the requirements of Annex $A$ of the Standard and would therefore require a site calibration. Table 3 shows the results from the site assessment, which confirm that a site calibration was not required.

Table 3. Criteria for test site without site calibration

\begin{tabular}{|l|c|c|c|c|}
\hline \multicolumn{1}{|c|}{ Description } & Distance & $\begin{array}{c}\text { Sector } \\
(\mathrm{deg})\end{array}$ & $\begin{array}{c}\text { Test Site } \\
\text { Condition }\end{array}$ & Pass/Fail \\
\hline Maximum slope of best fit plane $<3 \%$ & $<2 \mathrm{~L}$ & 360 & $2.9 \%$ & Pass \\
\hline $\begin{array}{l}\text { Maximum variation from best fit plane } \\
<0.08 \mathrm{D}\end{array}$ & $<2 \mathrm{~L}$ & 360 & 0.01 & Pass \\
\hline Maximum slope of best fit plane $<5 \%$ & $2-4 \mathrm{~L}$ & $\mathrm{In}$ & $1.2 \%$ & Pass \\
\hline $\begin{array}{l}\text { Maximum variation from best fit plane } \\
<0.15 \mathrm{D}\end{array}$ & $2-4 \mathrm{~L}$ & $\mathrm{In}$ & 0.02 & Pass \\
\hline Steepest slope maximum $<10 \%$ & $2-4 \mathrm{~L}$ & Out & $2.3 \%$ & Pass \\
\hline Maximum slope of best fit plane $<10 \%$ & $4-8 \mathrm{~L}$ & $\mathrm{In}$ & $1.2 \%$ & Pass \\
\hline $\begin{array}{l}\text { Maximum variation from best fit plane } \\
<0.15 \mathrm{D}\end{array}$ & $4-8 \mathrm{~L}$ & $\mathrm{In}$ & 0.02 & Pass \\
\hline No neighboring and operating turbines & $<2 \mathrm{D}_{\mathrm{n}}$ & 360 & 0 & Pass \\
\hline No obstacles & $<2 \mathrm{D}_{\mathrm{e}}$ & 360 & 0 & Pass \\
\hline
\end{tabular}

$\mathrm{D}=$ test turbine rotor diameter

$\mathrm{L}=$ distance between test turbine and meteorological tower

$\mathrm{D}_{\mathrm{e}}=$ equivalent diameter of obstacle

In = inside preliminary measurement sector

Out $=$ outside preliminary measurement sector

The Gaia-Wind 11-kW was connected to the electrical grid at a nominal voltage of $480 \mathrm{VAC}$ at a frequency of $60 \mathrm{~Hz}$. The grid tolerances are $5 \%$ for voltage amplitude and $1 \%$ for frequency. 


\section{Description of Test Equipment}

All test equipment was calibrated; Appendix B contains the calibration sheets. Table 4 shows the equipment used and calibration due dates. Figure 4 depicts the placement of the meteorological instruments on the tower. The primary anemometer was sent out for recalibration after the test period. The difference between the pre-test and post-test calibrations was within the tolerances allowed by the Standard.

Table 4. Equipment used in the power performance test

\begin{tabular}{|c|c|c|c|}
\hline Instrument & Make and Model & Serial Number & Calibration Due Date \\
\hline Power transducer & Ohio Semitronics, DMT 1040E & 06091046 & February 15,2010 \\
\hline Current transformers & Ohio Semitronics, 12974 & $\begin{array}{l}001293045 \\
001235428 \\
001293049\end{array}$ & $\begin{array}{l}\text { Calibrated with power } \\
\text { transducer }\end{array}$ \\
\hline Primary anemometer & Thies, First Class & 0707890 & April 7, 2009 \\
\hline Reference anemometer & NRG, Max 40 & 179500049023 & In situ \\
\hline Wind vane & $\begin{array}{l}\text { Met One, 020C with Aluminum } \\
\text { Vane }\end{array}$ & X4357 & April 7, 2009 \\
\hline $\begin{array}{l}\text { Pressure sensor } \\
\text { (replaced during test) }\end{array}$ & Vaisala, PTB101B & $\begin{array}{l}\text { C1040014 } \\
\text { T5030003 }\end{array}$ & $\begin{array}{l}\text { October 29, } 2008 \\
\text { August, } 262009\end{array}$ \\
\hline $\begin{array}{l}\text { Temperature sensor } \\
\text { (replaced during test) }\end{array}$ & Met One, T200 & $\begin{array}{l}0890084 \\
0789021\end{array}$ & $\begin{array}{l}\text { October 29, } 2008 \\
\text { October 10, } 2009\end{array}$ \\
\hline Precipitation sensor & Campbell Scientific, 237 & None & In situ \\
\hline $\begin{array}{l}\text { Data acquisition } \\
\text { system }\end{array}$ & $\begin{array}{l}\text { Compact DAQ w/LabView } \\
\text { cDAQ backplane } \\
\text { NI } 9229 \\
\text { NI } 9217 \\
\text { NI } 9205\end{array}$ & $\begin{array}{l}\text { 12E4DA3 } \\
\text { 12CBC7A } \\
\text { 12BFEE2 } \\
\text { 12E9C99 }\end{array}$ & $\begin{array}{l}\text { August 14, } 2008 \\
\text { July } 20,2008 \\
\text { October } 8,2008 \\
\text { Modules post-test } \\
\text { calibrated on May 5, } \\
2009, \text { were in } \\
\text { compliance }\end{array}$ \\
\hline
\end{tabular}




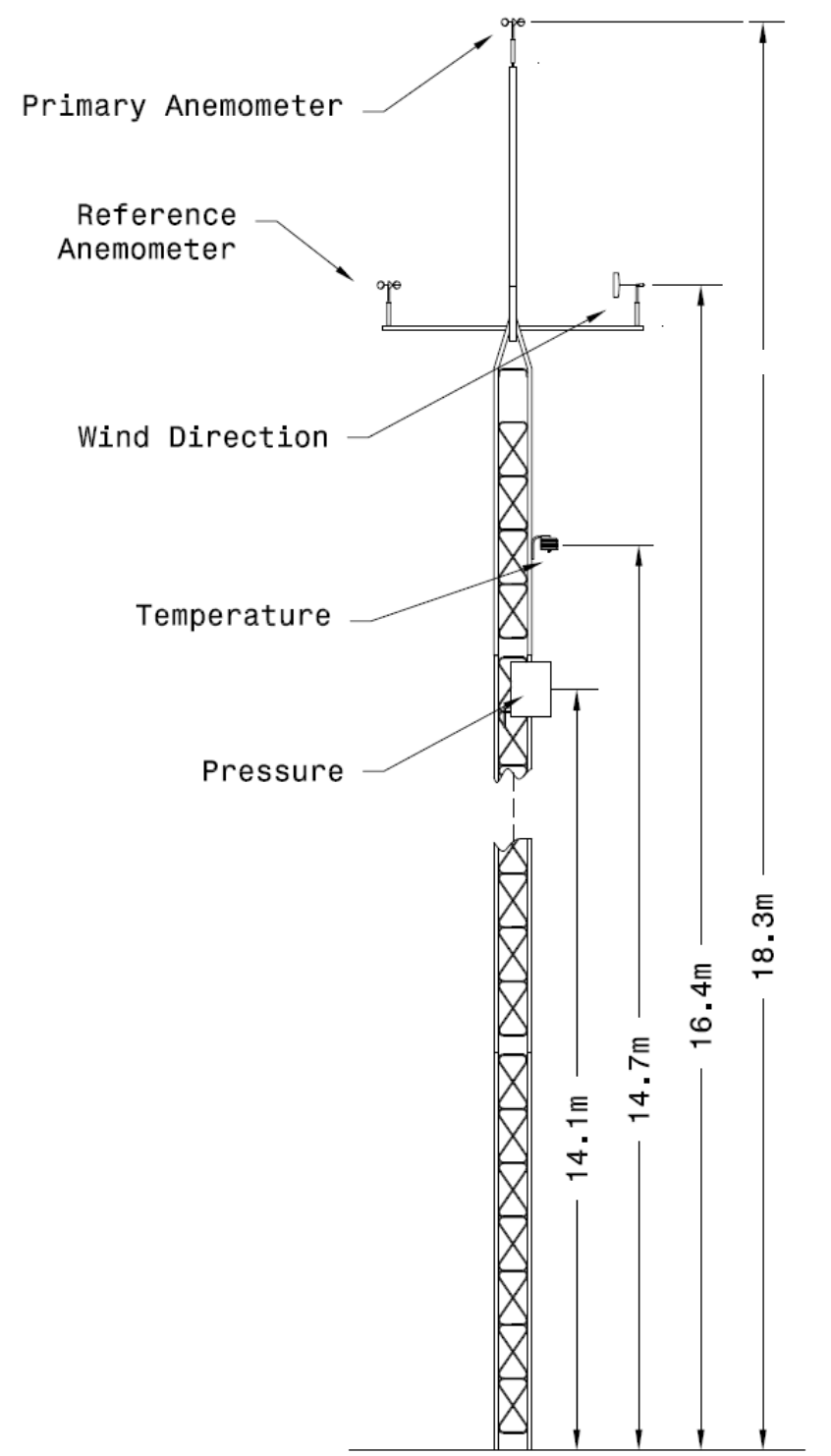

Figure 4. Meteorological tower and instruments

To ensure that only data obtained during normal operation of the turbine are used in the analysis, and to ensure that data are not corrupted, NREL excluded data sets from the database under the following circumstances:

- External conditions other than wind speed are out of the normal range for turbine operation.

- The turbine cannot operate because of a turbine fault condition.

- The turbine is manually shut down or in a test or maintenance operating mode.

Two methods are used to track when any of these conditions occur during the test. In the first method, the logbook will be checked for such events. The turbine controller provides two status signals that indicate when the turbine is available or braked. In the second method, these status signals are checked in the data file during analysis. 


\section{Description of Test Procedure}

NREL conducted the test according to the procedures in the Standard. The sampling rate was $10 \mathrm{kHz}$, decimated to $40 \mathrm{~Hz}$. The averaging time was 1 minute for the mean values. NREL also collected standard deviation, minimum, and maximum values for each averaging period.

The turbine status signals for the Gaia-Wind 11-kW originate from the turbine controller. The status signals indicated whether the turbine was available or braked.

Only database A is reported because the turbine did not reach cut-out wind speeds during the test period.

Table 5 gives the uncertainty sources and values used in the analysis.

Table 5. Uncertainty values used in the analysis

\begin{tabular}{|c|c|c|}
\hline Component & Uncertainty & Source \\
\hline \multicolumn{3}{|l|}{ Power } \\
\hline Current sensor/signal conditioner & $15.36 \mathrm{~W}$ & Specifications \\
\hline Power transducer & $0.09 \%$ & Specifications \\
\hline Data acquisition & $56 W+0.08 \%$ & Specifications \\
\hline Resistor & $0.01 \%$ & Specifications \\
\hline \multicolumn{3}{|l|}{ Wind Speed } \\
\hline Calibration & $0.01 \mathrm{~m} / \mathrm{s}$ & Calibration sheet \\
\hline Operational characteristics & $0.05 \mathrm{~m} / \mathrm{s}+0.52 \%$ & IEC equation (I.2) \\
\hline Mounting effects & $1.00 \%$ & Assumption \\
\hline Terrain effects & $2.00 \%$ & IEC \\
\hline Data acquisition & $<0.01 \mathrm{~m} / \mathrm{s}$ & Assumption \\
\hline \multicolumn{3}{|l|}{ Temperature } \\
\hline Temperature sensor & $0.15^{\circ} \mathrm{C}$ & Specifications \\
\hline Radiation shielding & $1.15^{\circ} \mathrm{C}$ & Assumption \\
\hline Mounting effects & $0.06{ }^{\circ} \mathrm{C}$ & IEC method \\
\hline Data acquisition & $0.35^{\circ} \mathrm{C}$ & Specifications \\
\hline \multicolumn{3}{|l|}{ Air Pressure } \\
\hline Pressure sensor & $0.20 \mathrm{kPa}$ & Specifications \\
\hline Mounting effects & $<0.01 \mathrm{kPa}$ & IEC method \\
\hline Data acquisition & $0.06 \mathrm{kPa}$ & Specifications \\
\hline
\end{tabular}




\section{Test Results}

\subsection{Tabular Results of Power Performance Test}

Table 6 through Table 9 list the power performance test results. Table 6 shows the binned power performance results at sea-level normalized air density. Table 7 shows the binned power performance results at the site average air density for the NWTC. 
Table 6. Performance at sea-level air density, $1.225 \mathrm{~kg} / \mathrm{m}^{3}$

\begin{tabular}{|c|c|c|c|c|c|c|c|}
\hline \multicolumn{8}{|c|}{ Measured Power Curve (Database A) } \\
\hline \multicolumn{5}{|c|}{ Reference Air Density: $1.225 \mathrm{~kg} / \mathrm{m}^{3}$} & \multirow{2}{*}{$\begin{array}{c}\text { Category A } \\
\text { Standard } \\
\text { Uncertainty } \\
\text { (kW) }\end{array}$} & \multirow{2}{*}{$\begin{array}{c}\text { Category B } \\
\text { Standard } \\
\text { Uncertainty } \\
\text { (kW) }\end{array}$} & \multirow{2}{*}{$\begin{array}{c}\text { Combined } \\
\text { Standard } \\
\text { Uncertainty } \\
\text { (kW) }\end{array}$} \\
\hline $\begin{array}{c}\text { Bin } \\
(\mathrm{m} / \mathrm{s})\end{array}$ & $\begin{array}{c}\text { Wind } \\
\text { Speed } \\
(\mathrm{m} / \mathrm{s})\end{array}$ & $\begin{array}{c}\text { Normalized } \\
\text { Power } \\
\text { Output } \\
\text { (kW) }\end{array}$ & $\mathbf{C}_{\mathrm{p}}$ & $\begin{array}{l}\text { Number } \\
\text { of } \\
\text { 1-Minute } \\
\text { Data } \\
\text { Sets }\end{array}$ & & & \\
\hline 3 & 3.00 & -0.10 & -0.05 & 7347 & 0.00 & 0.06 & 0.06 \\
\hline 3.5 & 3.49 & -0.11 & -0.03 & 6270 & 0.00 & 0.06 & 0.06 \\
\hline 4 & 3.99 & 0.31 & 0.06 & 5193 & 0.01 & 0.11 & 0.11 \\
\hline 4.5 & 4.49 & 1.15 & 0.16 & 3951 & 0.01 & 0.20 & 0.20 \\
\hline 5 & 4.99 & 2.28 & 0.23 & 3021 & 0.02 & 0.29 & 0.29 \\
\hline 5.5 & 5.49 & 3.67 & 0.27 & 2381 & 0.03 & 0.38 & 0.38 \\
\hline 6 & 5.99 & 5.00 & 0.29 & 2017 & 0.03 & 0.40 & 0.40 \\
\hline 6.5 & 6.49 & 6.27 & 0.28 & 1706 & 0.03 & 0.41 & 0.41 \\
\hline 7 & 7.00 & 7.57 & 0.27 & 1455 & 0.03 & 0.44 & 0.44 \\
\hline 7.5 & 7.49 & 8.70 & 0.25 & 1358 & 0.03 & 0.42 & 0.42 \\
\hline 8 & 7.99 & 9.80 & 0.24 & 1142 & 0.03 & 0.43 & 0.43 \\
\hline 8.5 & 8.49 & 10.77 & 0.22 & 970 & 0.03 & 0.41 & 0.41 \\
\hline 9 & 9.00 & 11.67 & 0.20 & 873 & 0.03 & 0.39 & 0.39 \\
\hline 9.5 & 9.50 & 12.36 & 0.18 & 713 & 0.04 & 0.32 & 0.32 \\
\hline 10 & 10.00 & 13.12 & 0.16 & 604 & 0.04 & 0.37 & 0.37 \\
\hline 10.5 & 10.49 & 13.69 & 0.15 & 520 & 0.03 & 0.30 & 0.30 \\
\hline 11 & 11.00 & 14.15 & 0.13 & 417 & 0.04 & 0.25 & 0.25 \\
\hline 11.5 & 11.49 & 14.59 & 0.12 & 336 & 0.04 & 0.26 & 0.26 \\
\hline 12 & 12.00 & 14.80 & 0.11 & 317 & 0.04 & 0.15 & 0.15 \\
\hline 12.5 & 12.49 & 14.90 & 0.09 & 280 & 0.06 & 0.11 & 0.13 \\
\hline 13 & 13.00 & 15.00 & 0.08 & 225 & 0.05 & 0.11 & 0.12 \\
\hline 13.5 & 13.49 & 14.93 & 0.08 & 187 & 0.06 & 0.10 & 0.12 \\
\hline 14 & 13.99 & 14.80 & 0.07 & 180 & 0.07 & 0.13 & 0.15 \\
\hline 14.5 & 14.48 & 14.60 & 0.06 & 146 & 0.08 & 0.16 & 0.18 \\
\hline 15 & 15.00 & 14.40 & 0.05 & 117 & 0.10 & 0.17 & 0.19 \\
\hline 15.5 & 15.48 & 14.49 & 0.05 & 100 & 0.10 & 0.11 & 0.15 \\
\hline 16 & 16.03 & 14.42 & 0.04 & 87 & 0.09 & 0.10 & 0.14 \\
\hline 16.5 & 16.50 & 14.15 & 0.04 & 70 & 0.13 & 0.24 & 0.27 \\
\hline 17 & 16.99 & 14.24 & 0.04 & 66 & 0.10 & 0.12 & 0.16 \\
\hline 17.5 & 17.50 & 14.13 & 0.03 & 59 & 0.10 & 0.13 & 0.16 \\
\hline 18 & 17.99 & 14.08 & 0.03 & 53 & 0.10 & 0.10 & 0.14 \\
\hline 18.5 & 18.48 & 13.91 & 0.03 & 31 & 0.07 & 0.17 & 0.18 \\
\hline 19 & 18.99 & 14.00 & 0.03 & 25 & 0.08 & 0.12 & 0.14 \\
\hline 19.5 & 19.45 & 14.11 & 0.02 & 13 & 0.11 & 0.14 & 0.18 \\
\hline 20 & 19.92 & 13.74 & 0.02 & 10 & 0.10 & 0.38 & 0.39 \\
\hline
\end{tabular}


Table 7. Performance at site average density, $0.95 \mathrm{~kg} / \mathrm{m}^{3}$

\begin{tabular}{|c|c|c|c|c|c|c|c|}
\hline \multicolumn{8}{|c|}{ Measured Power Curve (Database A) } \\
\hline \multicolumn{5}{|c|}{ Reference Air Density: $0.95 \mathrm{~kg} / \mathrm{m}^{3}$} & Category A & Category B & Combined \\
\hline $\begin{array}{c}\text { Bin } \\
(\mathrm{m} / \mathrm{s})\end{array}$ & $\begin{array}{c}\text { Wind } \\
\text { Speed } \\
(\mathrm{m} / \mathrm{s})\end{array}$ & $\begin{array}{c}\text { Normalized } \\
\text { Power } \\
\text { Output } \\
\text { (kW) }\end{array}$ & $\mathrm{C}_{\mathrm{p}}$ & $\begin{array}{c}\text { Number } \\
\text { of } \\
\text { 1-Minute } \\
\text { Data } \\
\text { Sets }\end{array}$ & $\begin{array}{c}\text { Standard } \\
\text { Uncertainty } \\
\text { (kW) }\end{array}$ & $\begin{array}{c}\text { Standard } \\
\text { Uncertainty } \\
(\mathrm{kW})\end{array}$ & $\begin{array}{c}\text { Standard } \\
\text { Uncertainty } \\
(\mathbf{k W})\end{array}$ \\
\hline 3 & 3.00 & -0.08 & -0.04 & 7347 & 0.00 & 0.06 & 0.06 \\
\hline 3.5 & 3.49 & -0.08 & -0.03 & 6270 & 0.00 & 0.06 & 0.06 \\
\hline 4 & 3.99 & 0.24 & 0.06 & 5193 & 0.01 & 0.09 & 0.09 \\
\hline 4.5 & 4.49 & 0.89 & 0.16 & 3951 & 0.01 & 0.16 & 0.16 \\
\hline 5 & 4.99 & 1.77 & 0.23 & 3021 & 0.02 & 0.23 & 0.23 \\
\hline 5.5 & 5.49 & 2.84 & 0.27 & 2381 & 0.02 & 0.30 & 0.30 \\
\hline 6 & 5.99 & 3.88 & 0.29 & 2017 & 0.02 & 0.31 & 0.31 \\
\hline 6.5 & 6.49 & 4.86 & 0.28 & 1706 & 0.03 & 0.32 & 0.32 \\
\hline 7 & 7.00 & 5.87 & 0.27 & 1455 & 0.03 & 0.34 & 0.34 \\
\hline 7.5 & 7.49 & 6.75 & 0.25 & 1358 & 0.02 & 0.33 & 0.33 \\
\hline 8 & 7.99 & 7.60 & 0.24 & 1142 & 0.02 & 0.33 & 0.33 \\
\hline 8.5 & 8.49 & 8.35 & 0.22 & 970 & 0.02 & 0.32 & 0.32 \\
\hline 9 & 9.00 & 9.05 & 0.20 & 873 & 0.03 & 0.30 & 0.30 \\
\hline 9.5 & 9.50 & 9.59 & 0.18 & 713 & 0.03 & 0.25 & 0.25 \\
\hline 10 & 10.00 & 10.17 & 0.16 & 604 & 0.03 & 0.29 & 0.29 \\
\hline 10.5 & 10.49 & 10.62 & 0.15 & 520 & 0.03 & 0.24 & 0.24 \\
\hline 11 & 11.00 & 10.98 & 0.13 & 417 & 0.03 & 0.20 & 0.20 \\
\hline 11.5 & 11.49 & 11.32 & 0.12 & 336 & 0.03 & 0.20 & 0.21 \\
\hline 12 & 12.00 & 11.48 & 0.11 & 317 & 0.03 & 0.12 & 0.12 \\
\hline 12.5 & 12.49 & 11.56 & 0.09 & 280 & 0.05 & 0.09 & 0.11 \\
\hline 13 & 13.00 & 11.63 & 0.08 & 225 & 0.04 & 0.09 & 0.10 \\
\hline 13.5 & 13.49 & 11.58 & 0.07 & 187 & 0.05 & 0.09 & 0.10 \\
\hline 14 & 13.99 & 11.48 & 0.07 & 180 & 0.05 & 0.11 & 0.12 \\
\hline 14.5 & 14.48 & 11.33 & 0.06 & 146 & 0.06 & 0.13 & 0.15 \\
\hline 15 & 15.00 & 11.17 & 0.05 & 117 & 0.08 & 0.13 & 0.15 \\
\hline 15.5 & 15.48 & 11.23 & 0.05 & 100 & 0.08 & 0.10 & 0.12 \\
\hline 16 & 16.03 & 11.18 & 0.04 & 87 & 0.07 & 0.09 & 0.11 \\
\hline 16.5 & 16.50 & 10.97 & 0.04 & 70 & 0.10 & 0.19 & 0.21 \\
\hline 17 & 16.99 & 11.04 & 0.04 & 66 & 0.08 & 0.10 & 0.13 \\
\hline 17.5 & 17.50 & 10.96 & 0.03 & 59 & 0.08 & 0.10 & 0.13 \\
\hline 18 & 17.99 & 10.92 & 0.03 & 53 & 0.08 & 0.09 & 0.12 \\
\hline 18.5 & 18.48 & 10.79 & 0.03 & 31 & 0.05 & 0.14 & 0.14 \\
\hline 19 & 18.99 & 10.86 & 0.03 & 25 & 0.06 & 0.10 & 0.12 \\
\hline 19.5 & 19.45 & 10.94 & 0.02 & 13 & 0.09 & 0.12 & 0.15 \\
\hline 20 & 19.92 & 10.65 & 0.02 & 10 & 0.08 & 0.30 & 0.31 \\
\hline
\end{tabular}


Table 8 shows the annual energy production at sea-level normalized air density. Table 9 shows the annual energy production (AEP) at the site average air density at the NWTC.

Table 8. Annual energy production at sea-level density, $1.225 \mathrm{~kg} / \mathrm{m}^{3}$

\begin{tabular}{|c|c|c|c|c|c|}
\hline \multicolumn{6}{|c|}{ Estimated annual energy production, database A (all valid data) } \\
\hline & $\begin{array}{r}\text { Reference air } \\
\text { density: } \\
\text { Cut-out wind } \\
\text { speed: }\end{array}$ & $\begin{array}{l}1.225 \\
25.00\end{array}$ & $\begin{array}{l}\mathrm{kg} / \mathrm{m}^{\wedge} 3 \\
\mathrm{~m} / \mathrm{s}\end{array}$ & & \\
\hline $\begin{array}{l}\text { Hub height annual } \\
\text { average wind } \\
\text { speed (Rayleigh) }\end{array}$ & \multirow{2}{*}{$\begin{array}{c}\text { AEP-measured } \\
\text { kWh }\end{array}$} & \multicolumn{2}{|c|}{$\begin{array}{l}\text { Standard Uncertainty } \\
\text { in AEP-measured }\end{array}$} & \multirow{2}{*}{$\begin{array}{c}\text { AEP-extrapolated } \\
\text { kWh }\end{array}$} & \multirow[t]{2}{*}{$\begin{array}{c}\text { Complete if AEP } \\
\text { measured is at leas } \\
95 \% \text { of AEP } \\
\text { extrapolated }\end{array}$} \\
\hline $\mathrm{m} / \mathrm{s}$ & & kWh & $\%$ & & \\
\hline 4 & 17,716 & 1,693 & $10 \%$ & 17,716 & Complete \\
\hline 5 & 32,122 & 2,093 & $7 \%$ & 32,122 & Complete \\
\hline 6 & 46,292 & 2,284 & $5 \%$ & 46,313 & Complete \\
\hline 7 & 58,690 & 2,328 & $4 \%$ & 58,893 & Complete \\
\hline 8 & 68,525 & 2,286 & $3 \%$ & 69,394 & Complete \\
\hline 9 & 75,474 & 2,199 & $3 \%$ & 77,764 & Complete \\
\hline 10 & 79,617 & 2,089 & $3 \%$ & 84,067 & Incomplete \\
\hline 11 & 81,326 & 1,968 & $2 \%$ & 88,411 & Incomplete \\
\hline \multicolumn{6}{|c|}{$\begin{array}{l}\text { AEP measured assumes zero power between highest bin and cutout } \\
\text { EP extrapolated assumes power in last bin between last bin and cutout }\end{array}$} \\
\hline
\end{tabular}

Table 9. Annual energy production at site average density, $0.95 \mathrm{~kg} / \mathrm{m}^{3}$

\begin{tabular}{|c|c|c|c|c|c|}
\hline \multicolumn{6}{|c|}{ Estimated annual energy production, database A (all valid data) } \\
\hline \multirow{3}{*}{$\begin{array}{c}\text { Hub height annual } \\
\text { average wind } \\
\text { speed (Rayleigh) } \\
\mathrm{m} / \mathrm{s}\end{array}$} & $\begin{array}{l}\text { Reference air density: } \\
\text { Cut-out wind speed: }\end{array}$ & $\begin{array}{c}0.95 \\
25.00 \\
\end{array}$ & $\begin{array}{l}\mathrm{kg} / \mathrm{m}^{\wedge} 3 \\
\mathrm{~m} / \mathrm{s}\end{array}$ & & \\
\hline & \multirow{2}{*}{$\begin{array}{l}\text { AEP-measured } \\
\text { kWh }\end{array}$} & \multicolumn{2}{|c|}{$\begin{array}{c}\text { Standard } \\
\text { Uncertainty in } \\
\text { AEP-measured }\end{array}$} & \multirow{2}{*}{$\begin{array}{c}\text { AEP-extrapolated } \\
\text { kWh }\end{array}$} & \multirow[t]{2}{*}{$\begin{array}{c}\text { Complete if AEP } \\
\text { measured } \\
\text { is at least } 95 \% \text { of AEP } \\
\text { extrapolated }\end{array}$} \\
\hline & & kWh & $\%$ & & \\
\hline 4 & 13,739 & 1,381 & $10 \%$ & 13,739 & Complete \\
\hline 5 & 24,911 & 1,679 & $7 \%$ & 24,911 & Complete \\
\hline 6 & 35,900 & 1,820 & $5 \%$ & 35,916 & Complete \\
\hline 7 & 45,515 & 1,850 & $4 \%$ & 45,672 & Complete \\
\hline 8 & 53,142 & 1,816 & $3 \%$ & 53,816 & Complete \\
\hline 9 & 58,531 & 1,748 & $3 \%$ & 60,307 & Complete \\
\hline 10 & 61,744 & 1,661 & $3 \%$ & 65,195 & Incomplete \\
\hline 11 & 63,069 & 1,566 & $2 \%$ & 68,564 & Incomplete \\
\hline \multicolumn{6}{|c|}{$\begin{array}{l}\text { AEP measured assumes zero power between highest bin and cutout } \\
\text { EP extrapolated assumes power in last bin between last bin and cutout }\end{array}$} \\
\hline
\end{tabular}




\subsection{Graphical Results Power Performance Test}

Figure 5 through Figure 11 show the results of the power performance test. Figure 5 shows a plot of the binned power curve normalized to sea-level air density.

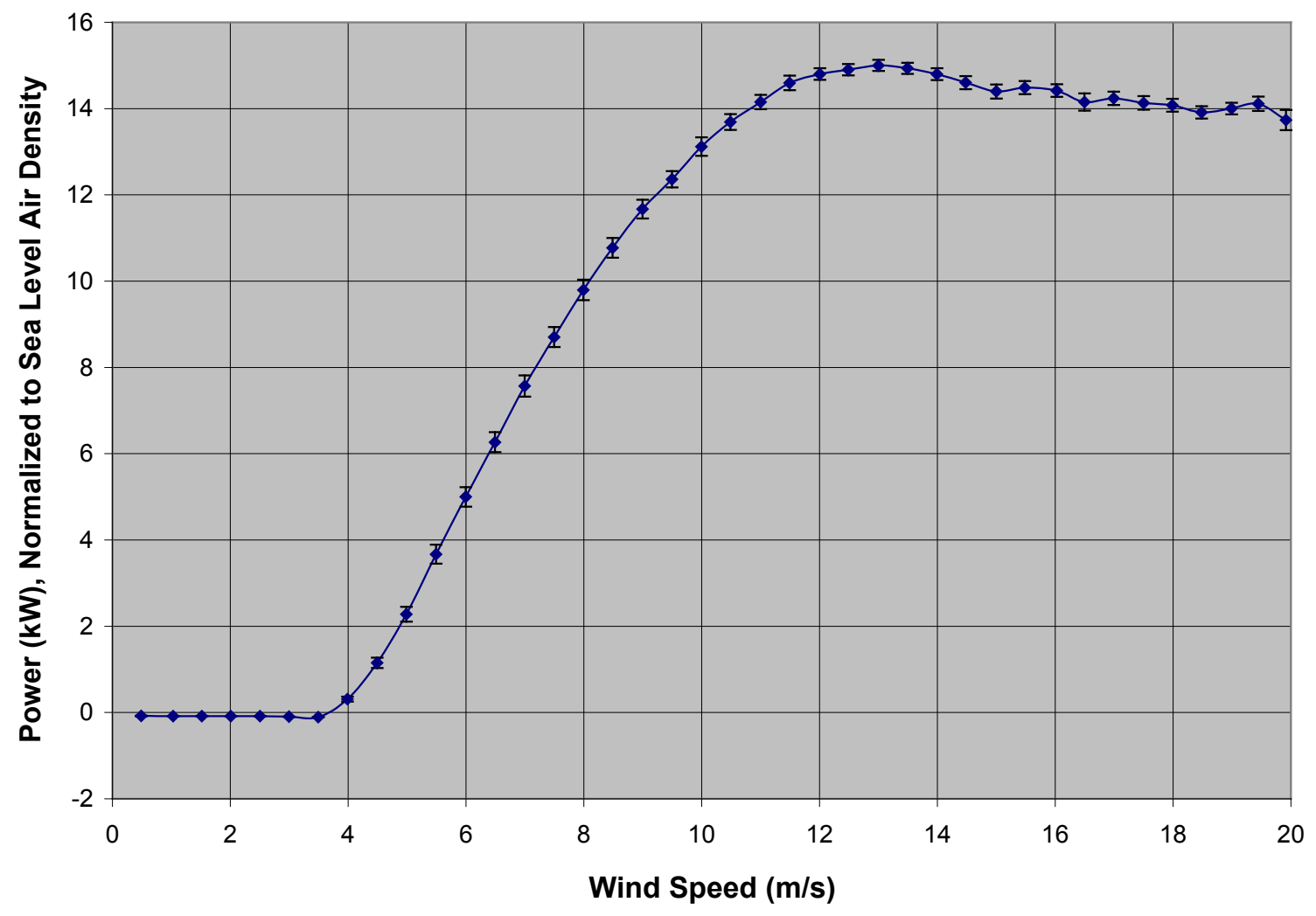

Figure 5. Power curve at sea-level density, $1.225 \mathrm{~kg} / \mathrm{m}^{3}$ 
Figure 6 shows a plot of the binned power curve at the site average air density during the test period.

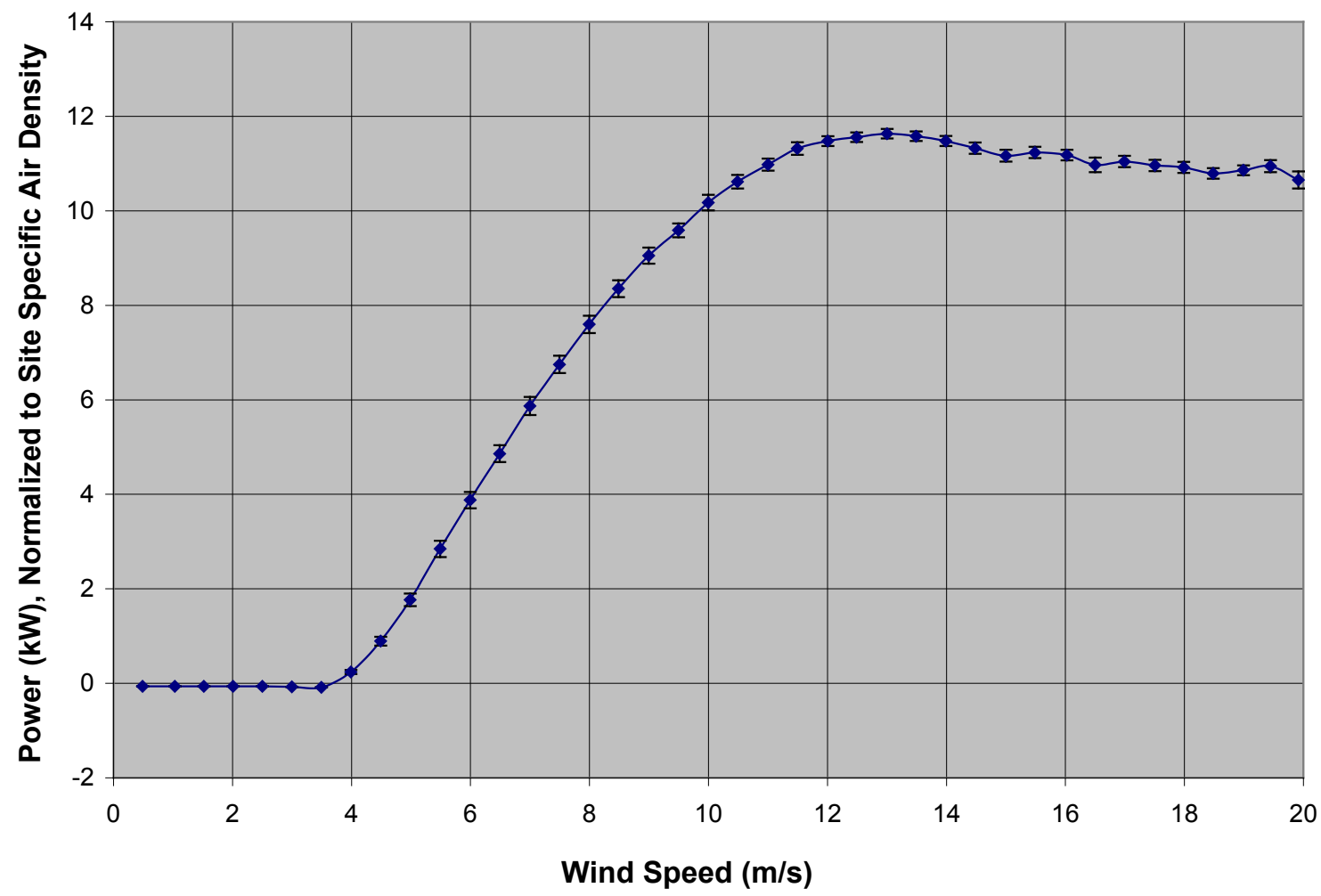

Figure 6. Power curve at site average density, $0.95 \mathrm{~kg} / \mathrm{m}^{3}$ 
Figure 7 shows a scatter plot of statistics for power for the turbine.

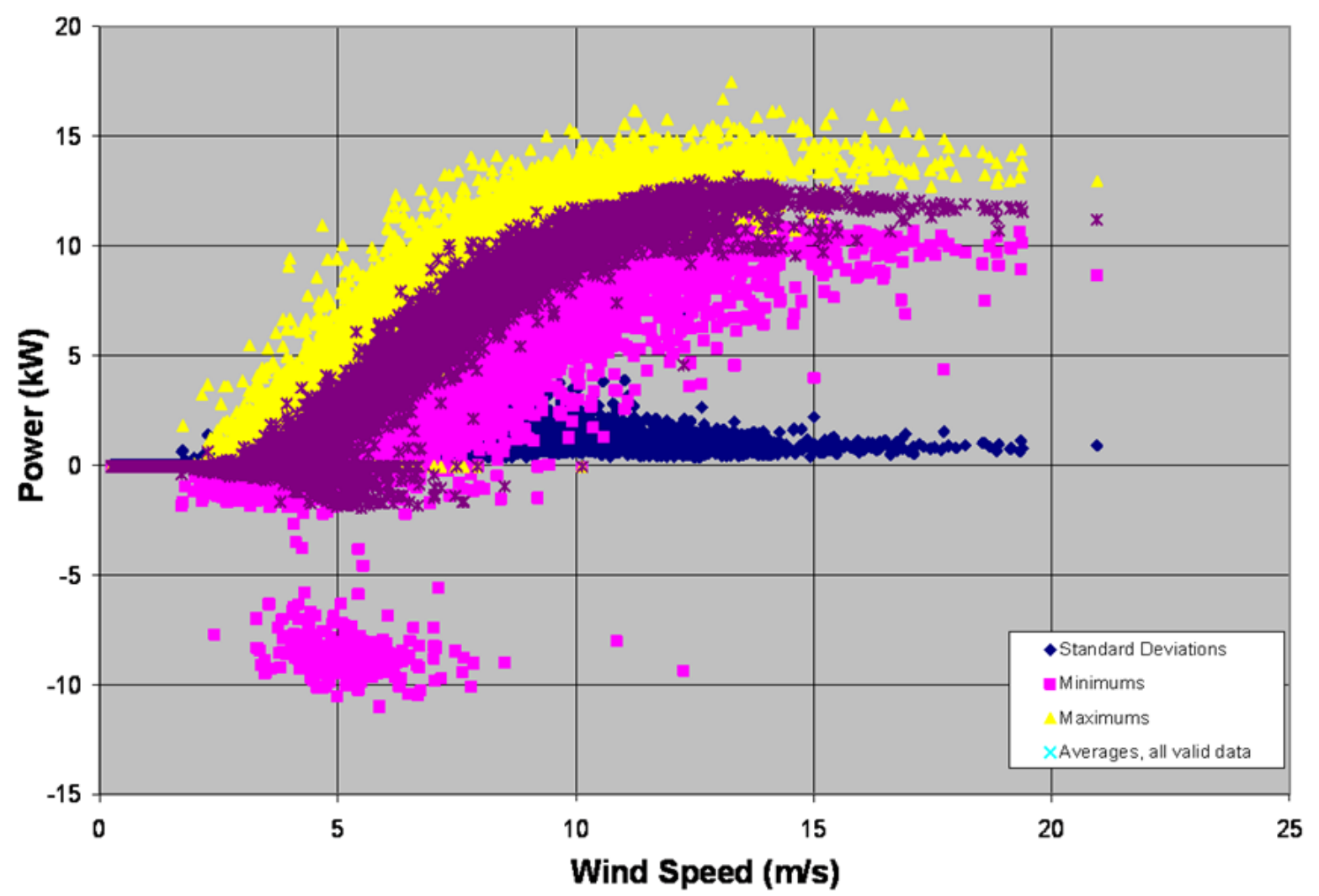

Figure 7. Scatter plot of mean, standard deviation, minimum, and maximum power data

Multiple power curves appear at the higher wind speeds. NREL investigated the cause but did not find it during testing. NREL changed two contactors in the controller during the test, but this did not alter the performance of the turbine. The contactor replacement did not cause the multiple power curves. 
Figure 8 shows a plot of the binned coefficient of performance as a function of wind speed at sea-level normalized air density.

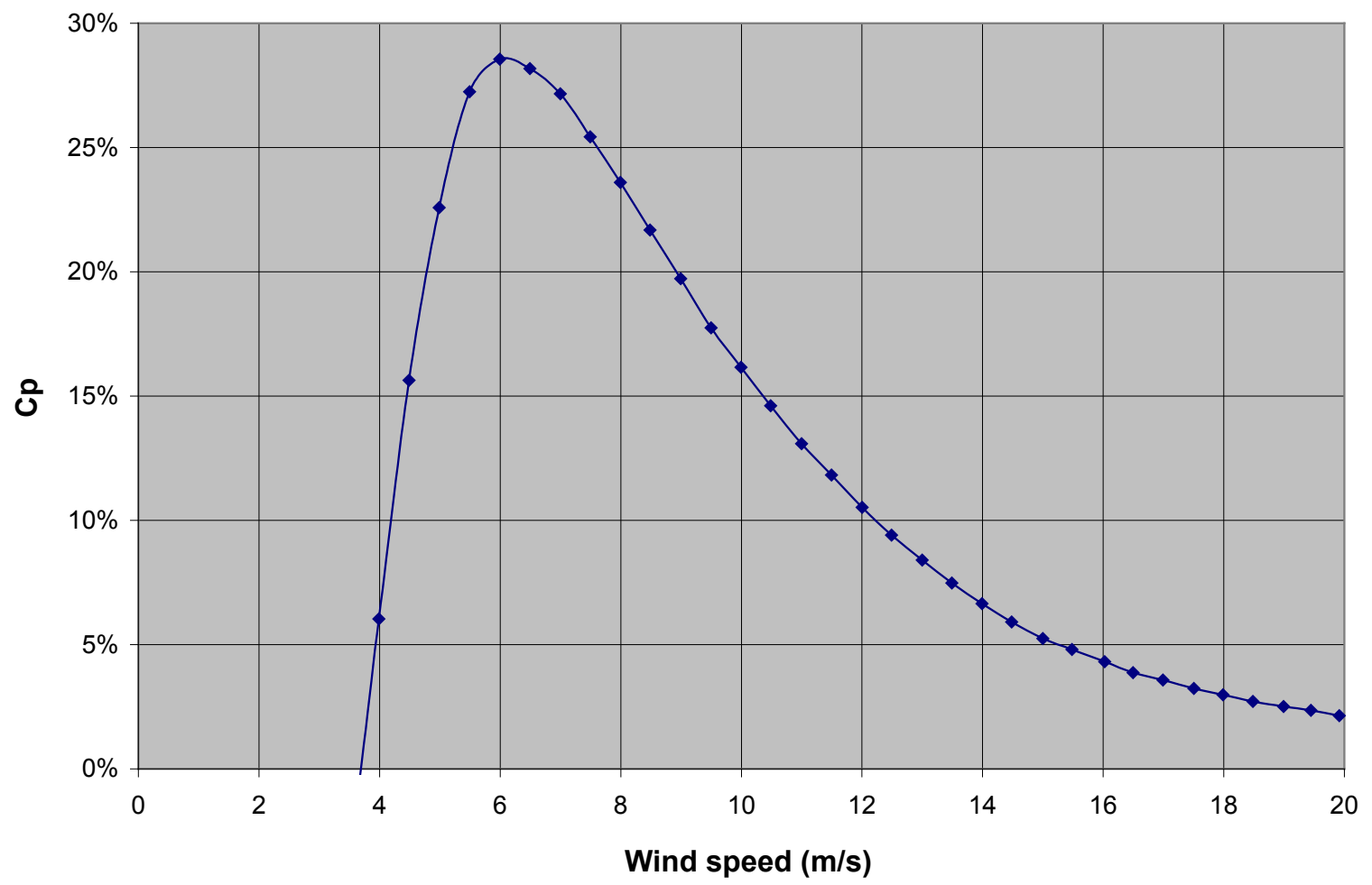

Figure 8. Coefficient of power at sea-level density, $1.225 \mathrm{~kg} / \mathrm{m}^{3}$ 
Figure 9 shows a scatter plot and binned turbulence intensity as a function of wind speed.

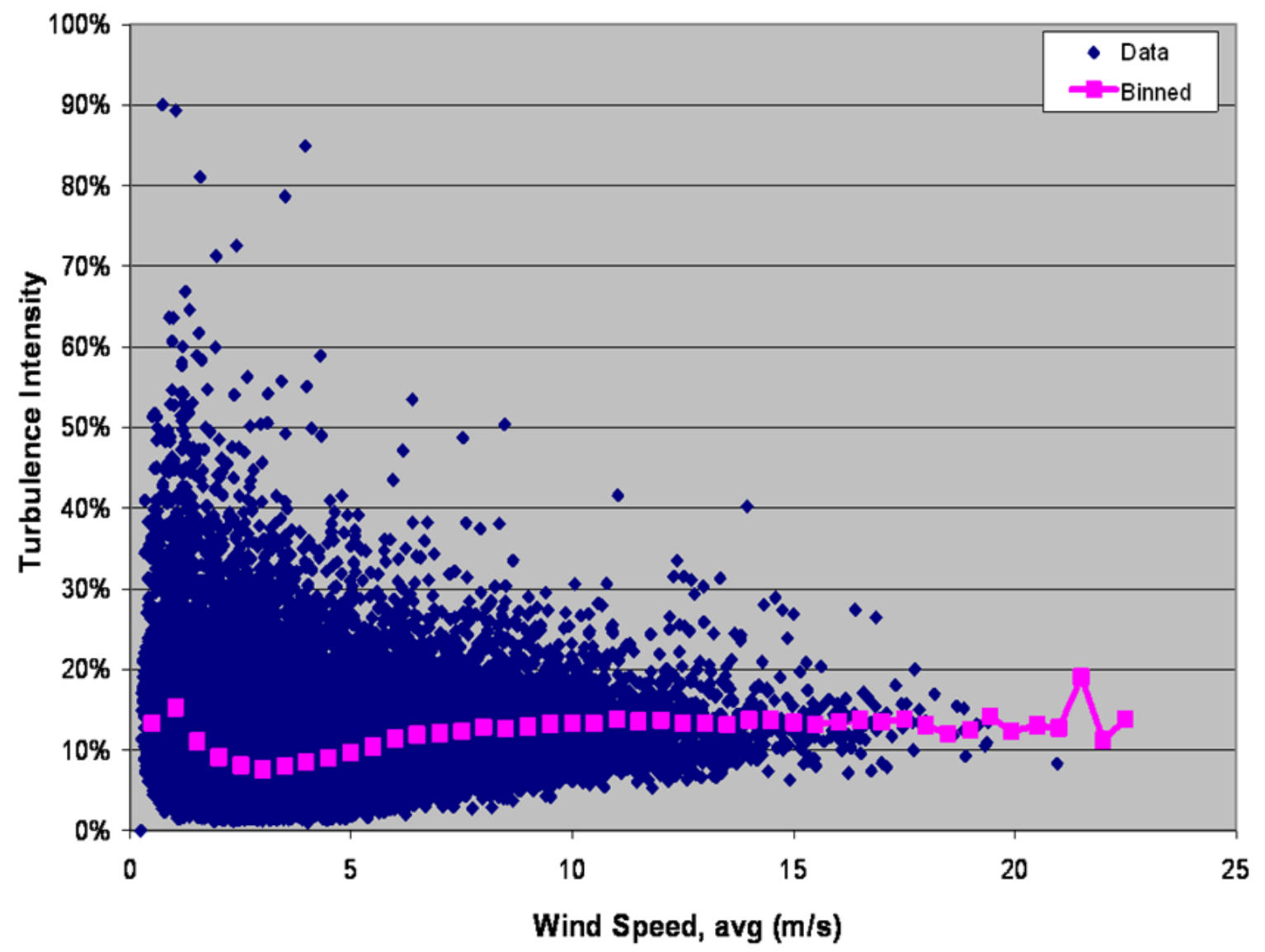

Figure 9. Wind turbulence intensity as a function of wind speed 
Figure 10 shows a scatter plot of wind speed and turbulence intensity as a function of wind direction.

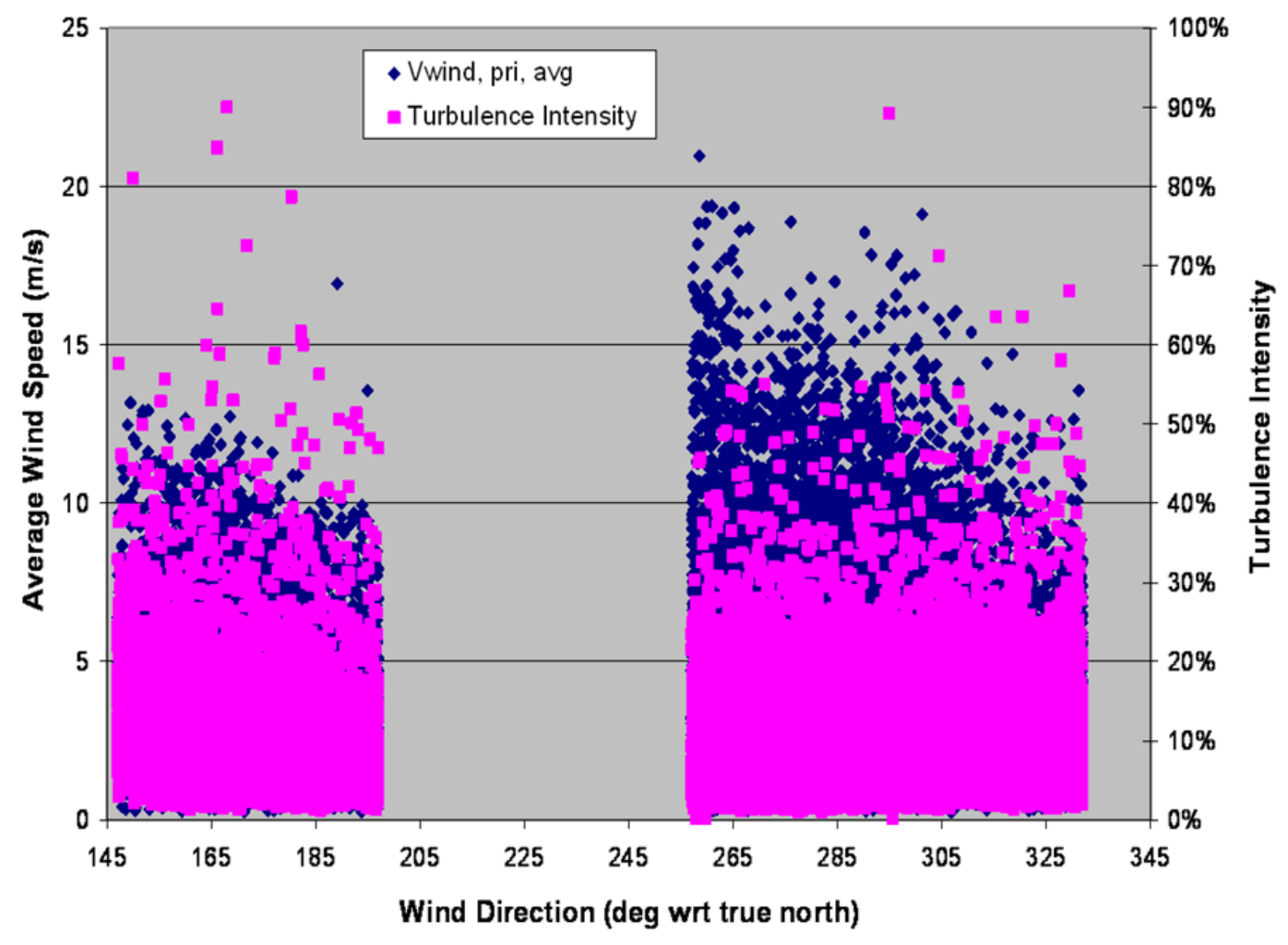

Figure 10. Wind speed and turbulence intensity as a function of wind direction for the measurement sector 
Figure 11 shows a scatter plot and binned values of rotor speed as a function of wind speed.

\begin{tabular}{|c|c|c|c|c|c|c|c|c|c|c|c|c|c|c|c|}
\hline Wind speed & $\mathrm{m} / \mathrm{s}$ & 0.5 & 1 & 1.5 & 2 & 2.5 & 3 & 3.5 & 4 & 4.5 & 5 & 5.5 & 6 & 6.5 & 7 \\
\hline Rotor speed & $\mathrm{rpm}$ & 0.3 & 1.4 & 3.8 & 8.5 & 13.6 & 20.8 & 30.7 & 37.4 & 45.3 & 51.9 & 57.1 & 59.0 & 59.4 & 60.2 \\
\hline & (o & & 8 & $8 \mathrm{O}$ & & 0 & & $10-$ & & $11 \mathrm{Cl}$ & 12 & $12 \mathrm{C}$ & إב & 135 & \\
\hline Rotor speed & rpm & 60.6 & 60.7 & 608 & 60.8 & 60.8 & 60.9 & 61.0 & 61.0 & 61.1 & 61.1 & 61.0 & 61.1 & 61.1 & 61.1 \\
\hline & & & & & & & & & & & & & & & \\
\hline Wind speed & $\mathrm{m} / \mathrm{s}$ & 14.5 & 15 & 15.5 & 16 & 16.5 & 17 & 17.5 & 18 & 18.5 & 19 & 19.5 & 20 & 20.5 & 21 \\
\hline Rotor speed & $\mathrm{rpm}$ & 61.1 & 61.1 & 61.1 & 61.1 & 61.1 & 610 & 610 & 610 & 610 & 610 & 611 & 610 & 610 & 611 \\
\hline
\end{tabular}

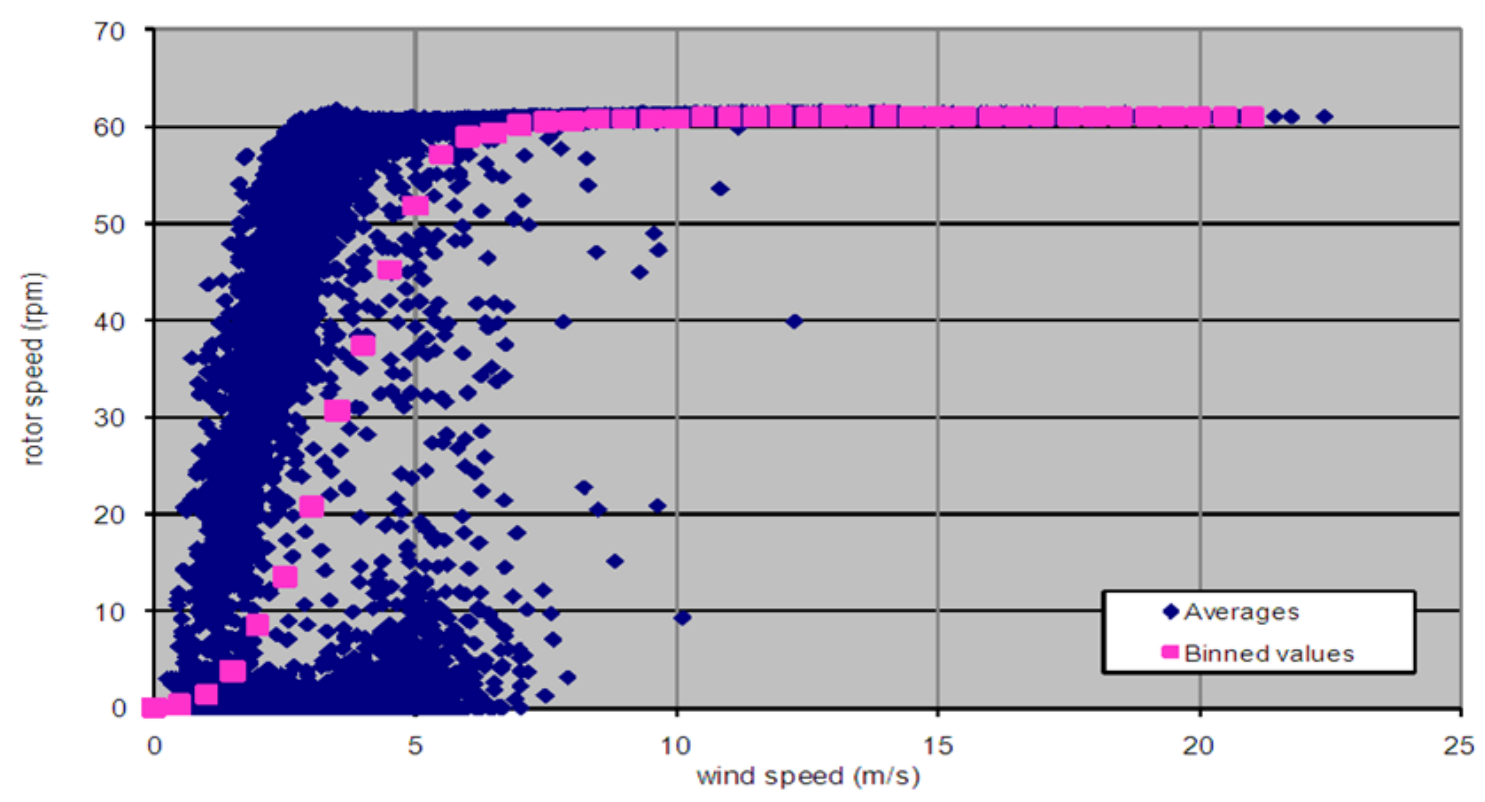

Figure 11. Rotor speed as a function of wind speed (1-minute averages) and binned values 


\section{Deviations and Exceptions}

\subsection{Deviations from the Standard}

The current transformers are not compliant with IEC 60044-1 but do exceed the minimum accuracy required by the standard.

\subsection{Exceptions to NWTC-CT Quality Assurance System}

The data acquisition modules were used beyond the calibration due date. The modules were post-test calibrated and found to be in compliance within the specifications. Appendix B includes the post-test calibration sheets. 
A. Appendix - Photographs of the Test Site from the Turbine Base 


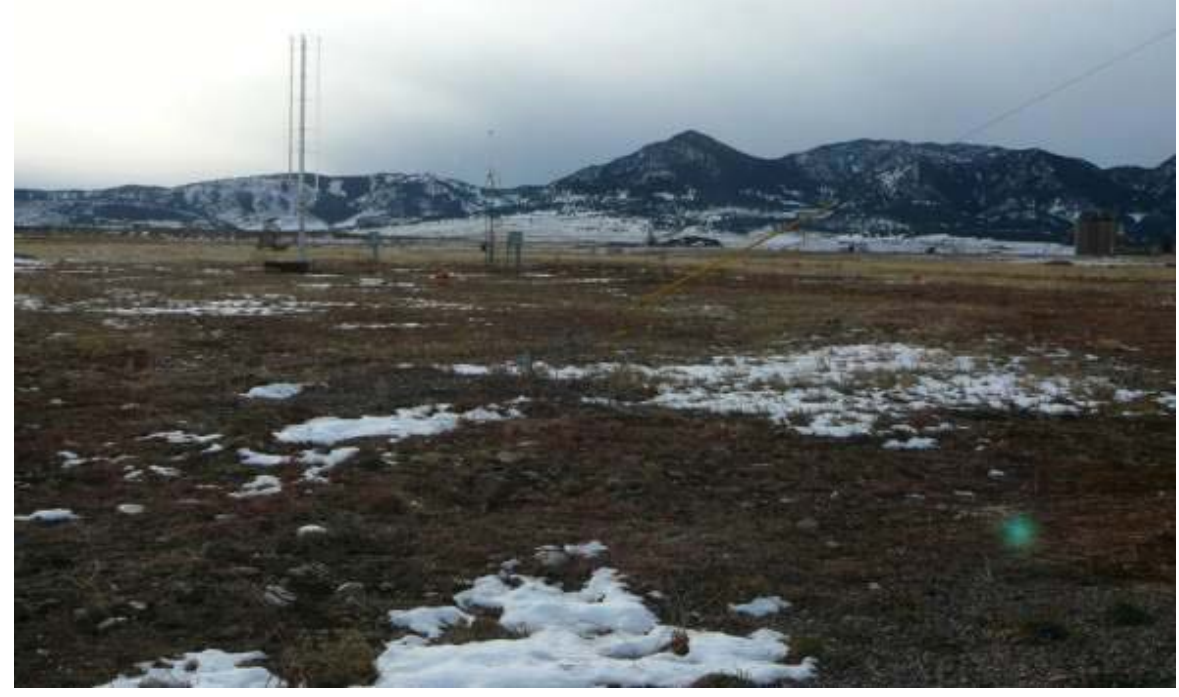

Figure A.1. Southwest

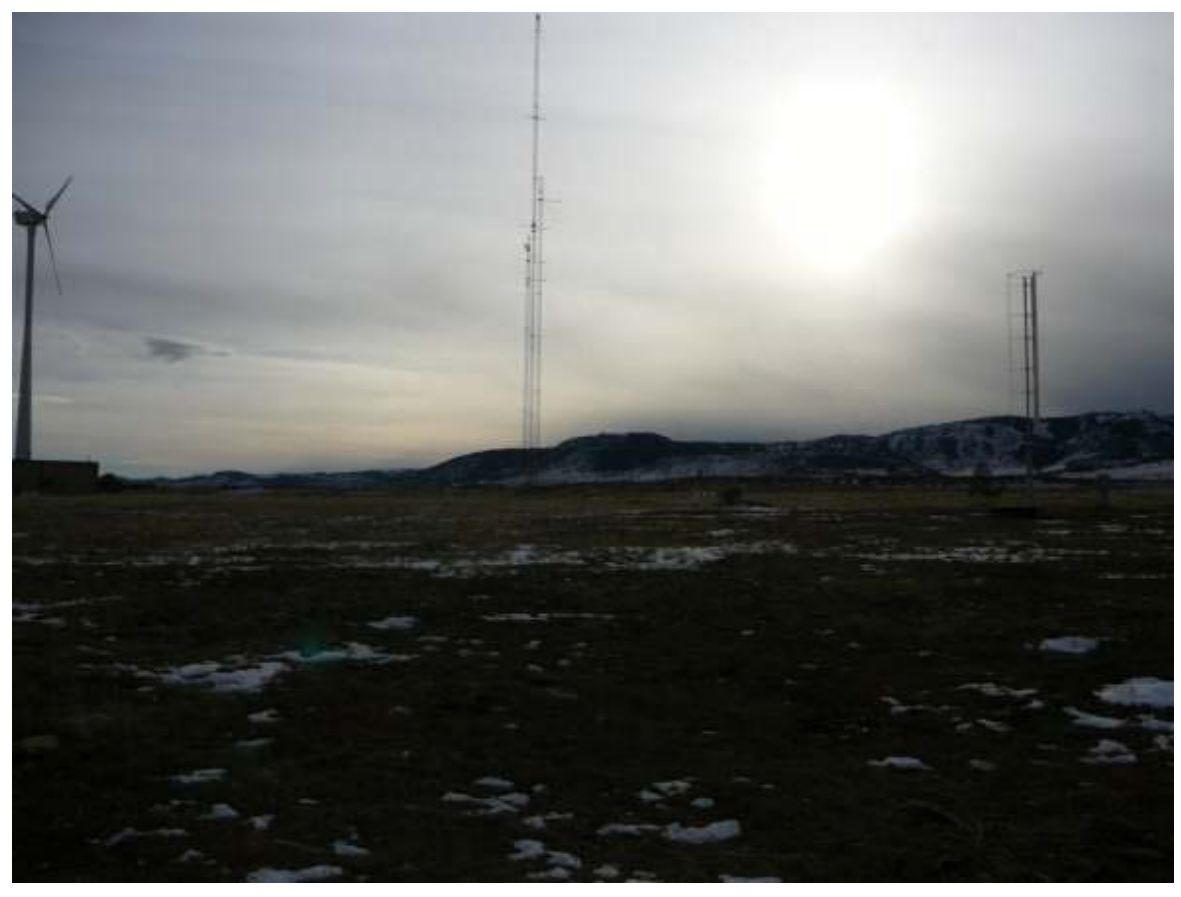

Figure A.2. South-southwest 


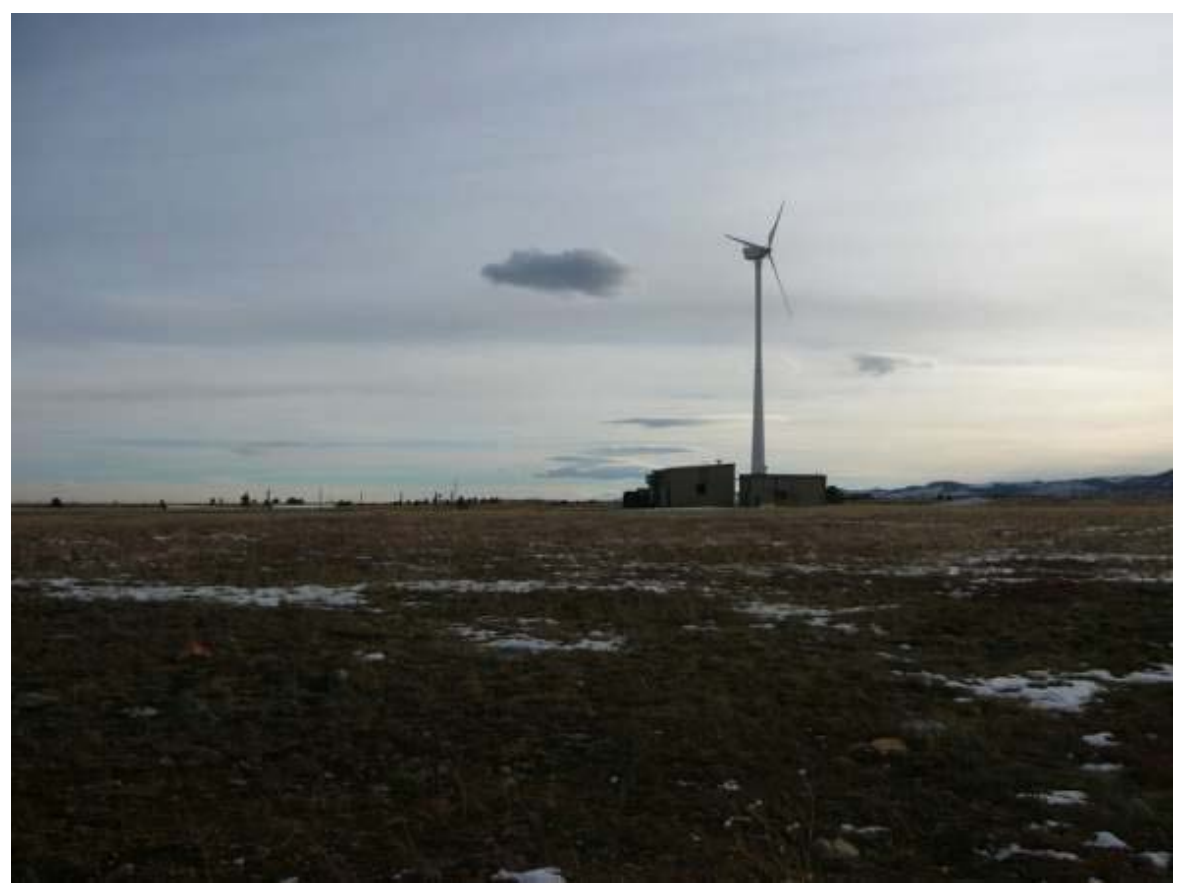

Figure A.3. South

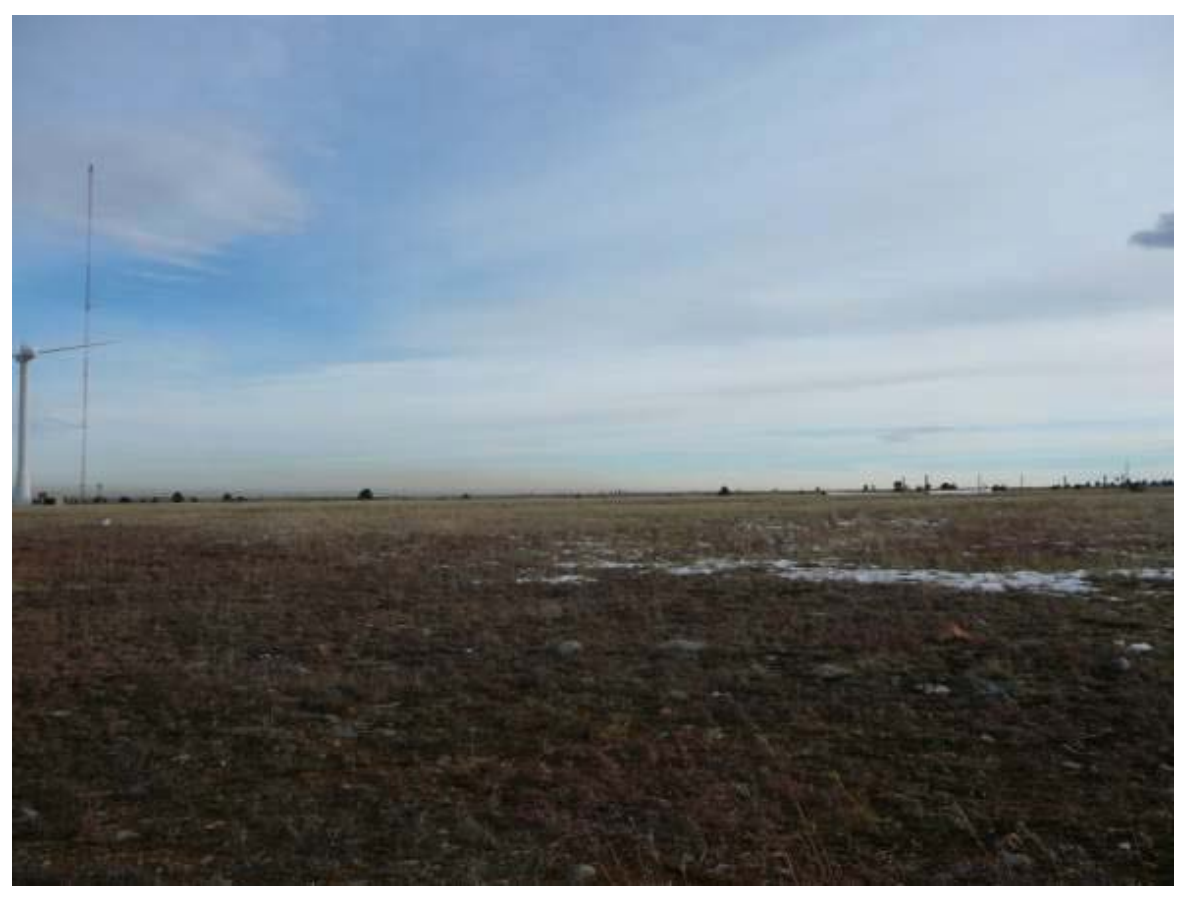

Figure A.4. Southeast 


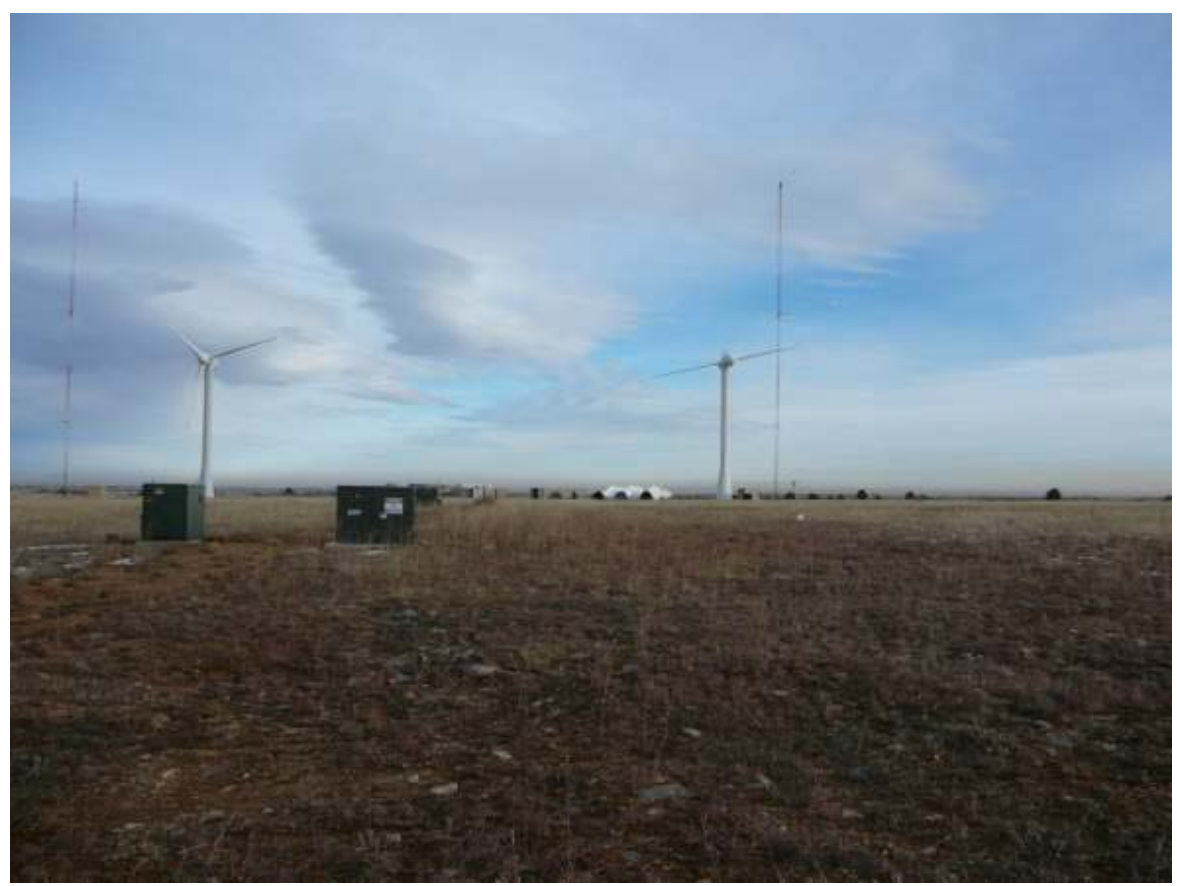

Figure A.5. East

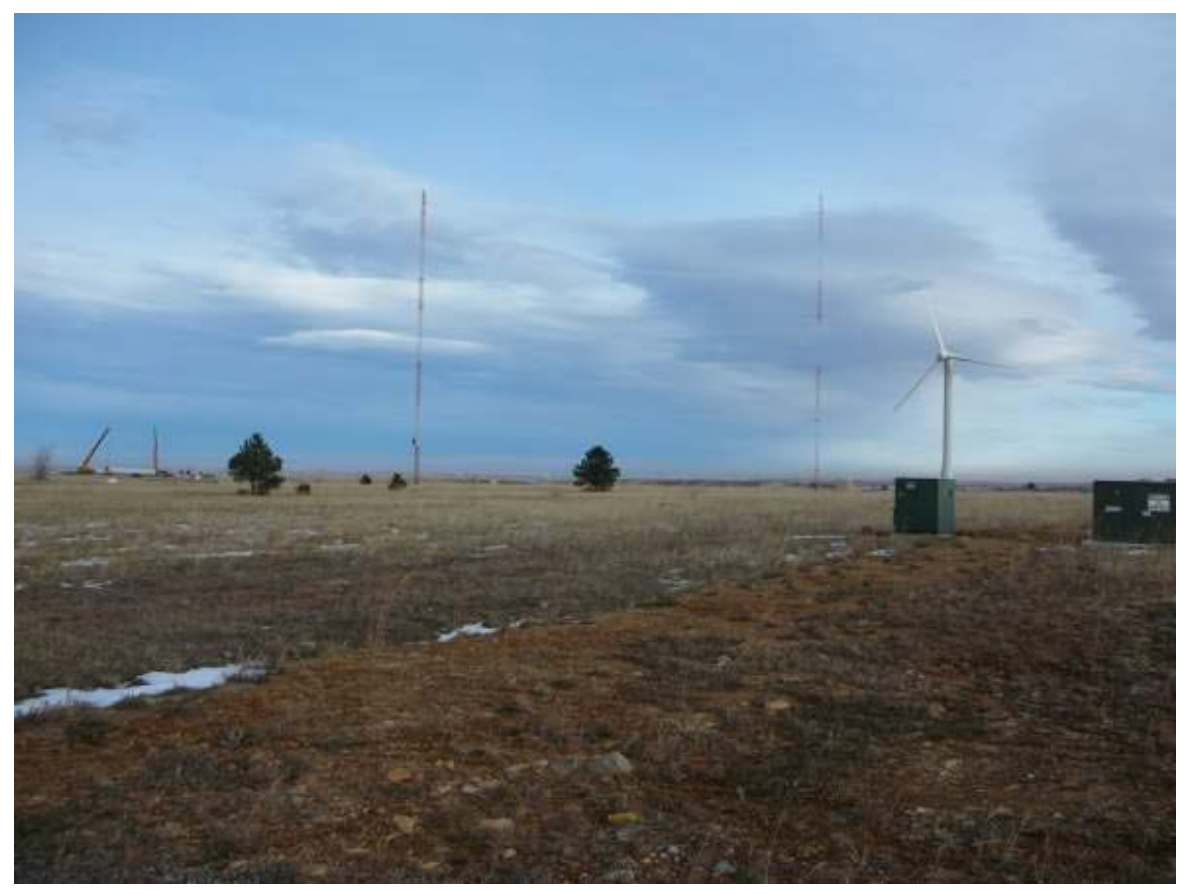

Figure A.6. East-northeast 


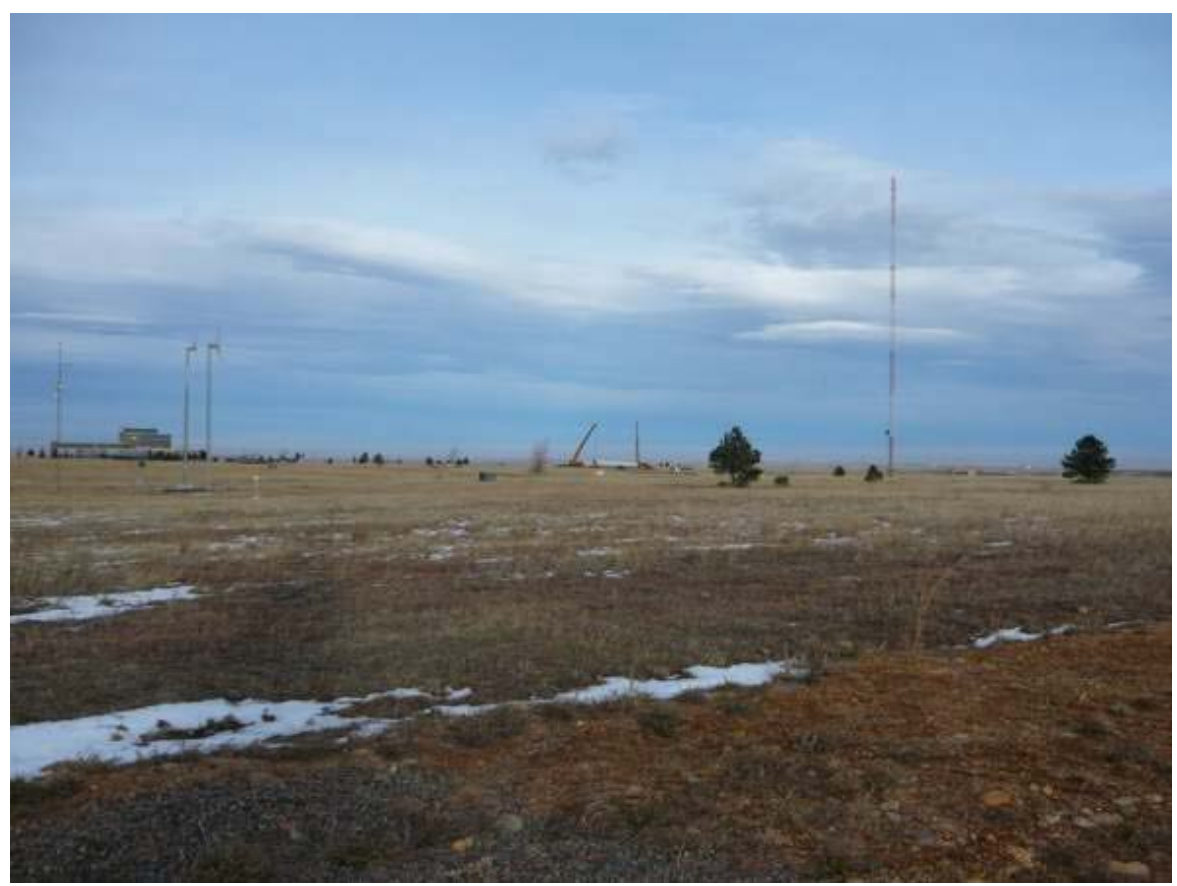

Figure A.7. Northeast

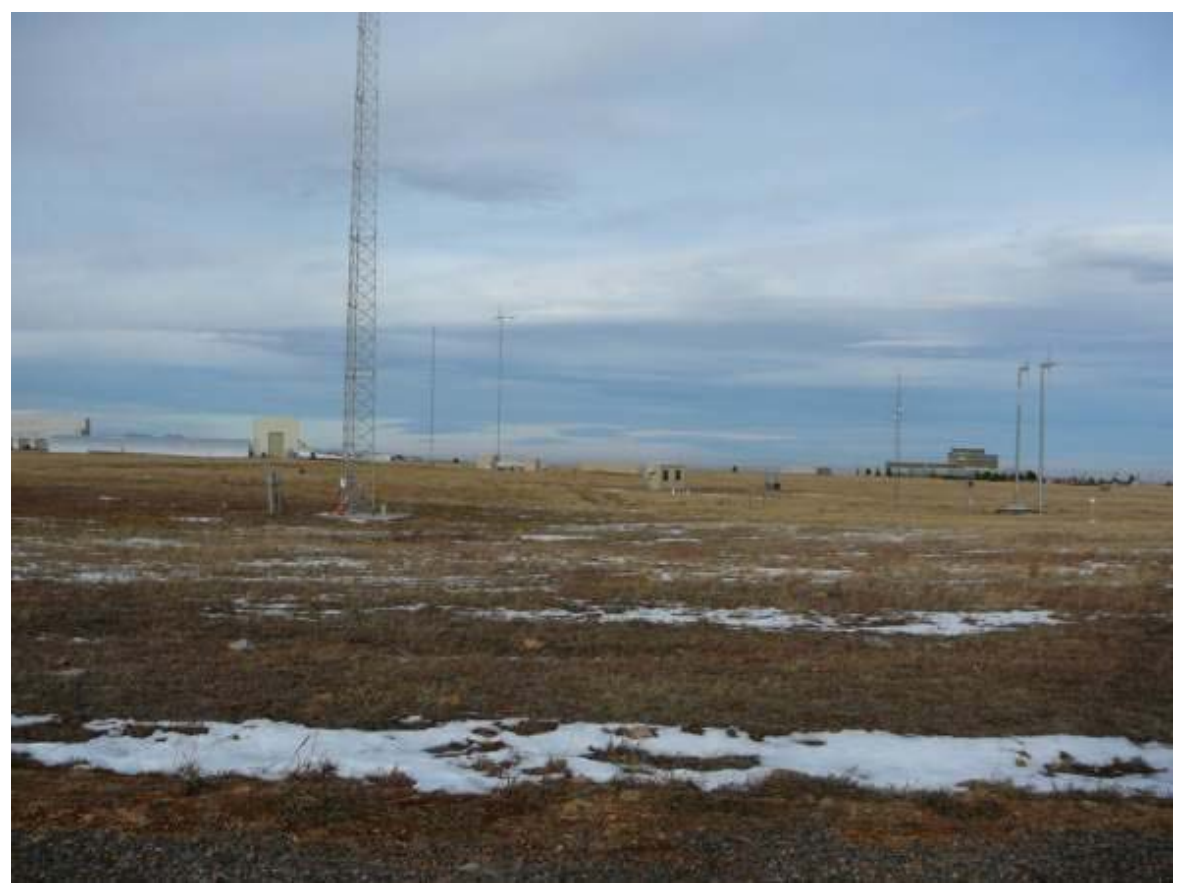

Figure 12. North 


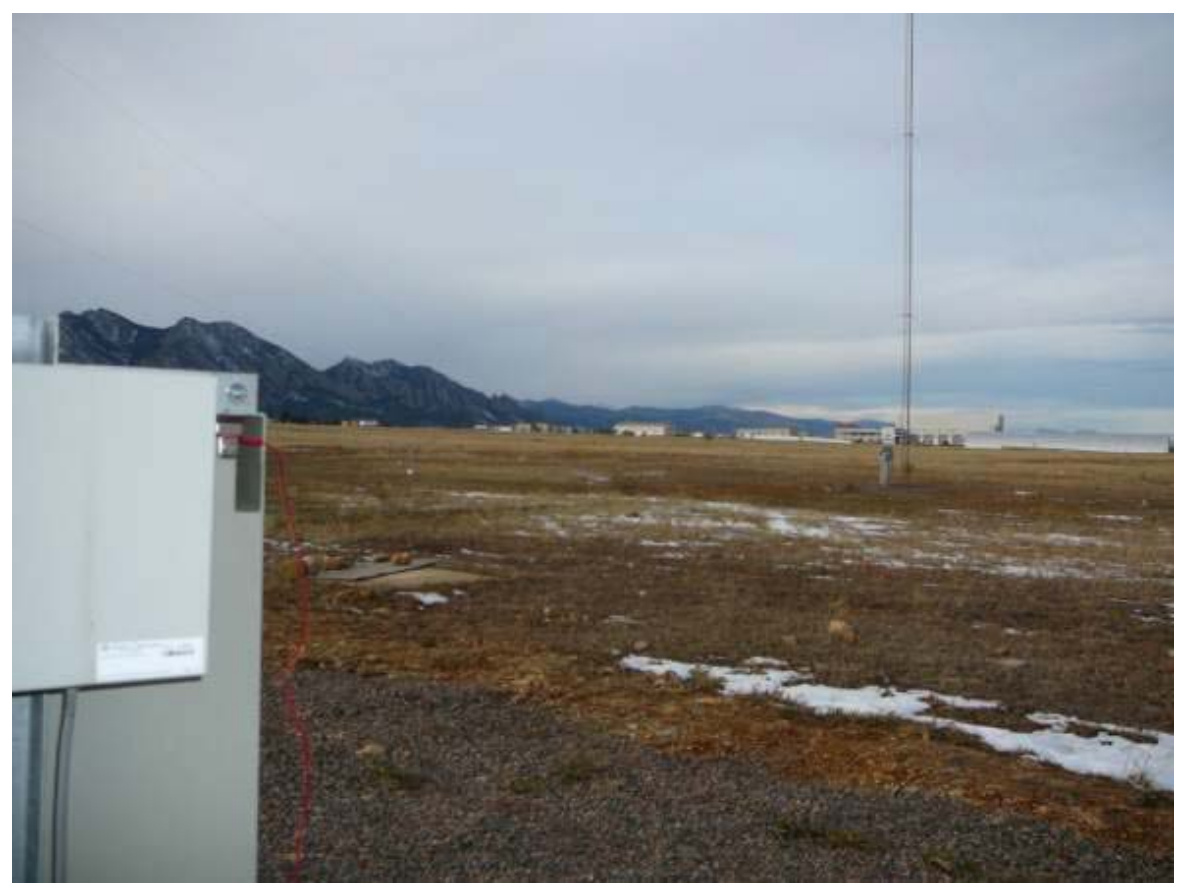

Figure A.9. Northwest

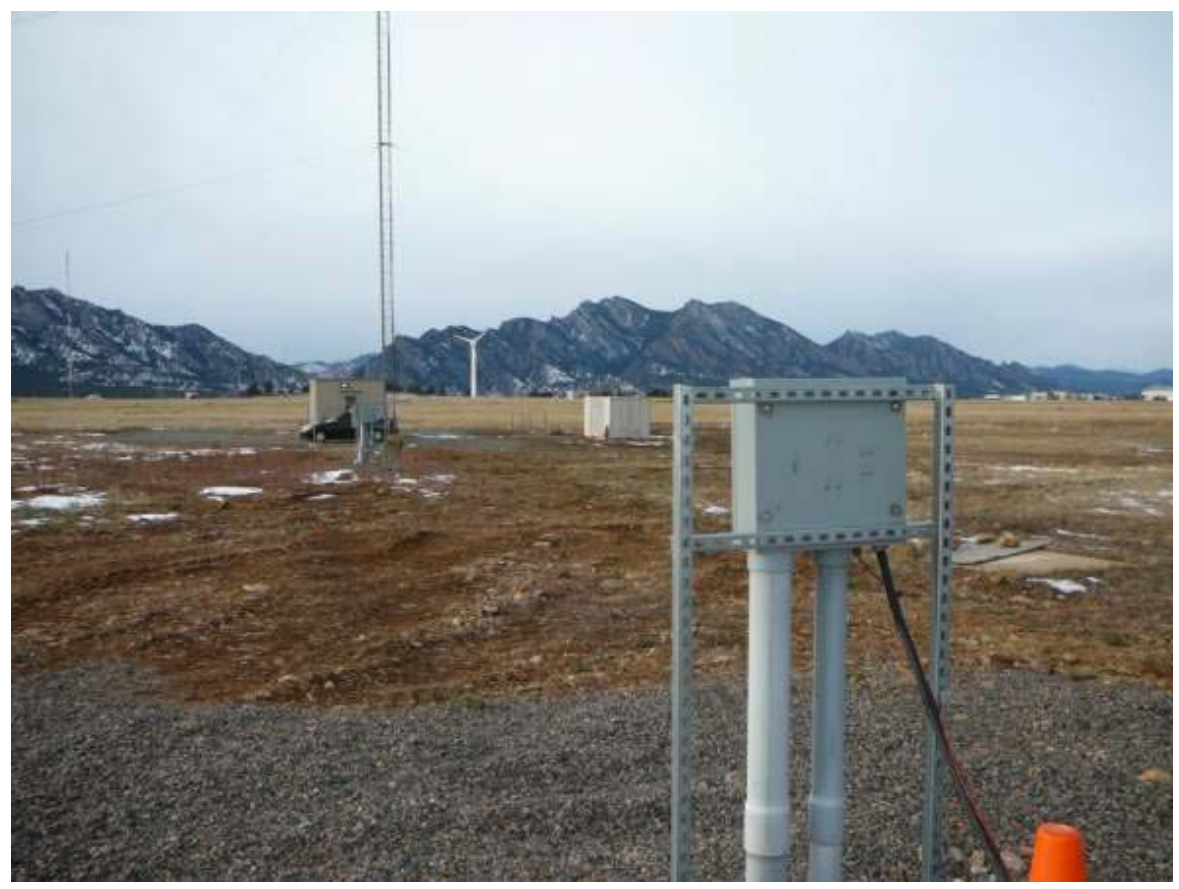

Figure A.10. West 


\section{B. Appendix - Equipment Calibration Sheets}

Branch \#: 5000

\section{NREL METROLOGY LABORATORY}

Test Report

Test Instrument: Multifunction Transduce

DOB \#: $03575 C$

Model \#

; $D M T-1040 E$

$S / N: 06091046$

Calibration Date: $02 / 15 / 2008$

Due Date: $02 / 15 / 2010$

A. Set-Up for Total Power Callbration:

A.1. Voltage is applied to Lines 1, 2, \& $3=277.128 \mathrm{~V}$ (1) $60 \mathrm{~Hz}$.

A.2. Current is applied to $n=8$-TURNS through three current transformers

that are connected to Lines $1,2, \& 3$.

A.3. Analog Output -1 is measured across precision resistor $=250 \Omega$.

A.4. Full Scale setting $=-15.796 \mathrm{KW}$ to $15.796 \mathrm{KW}$.

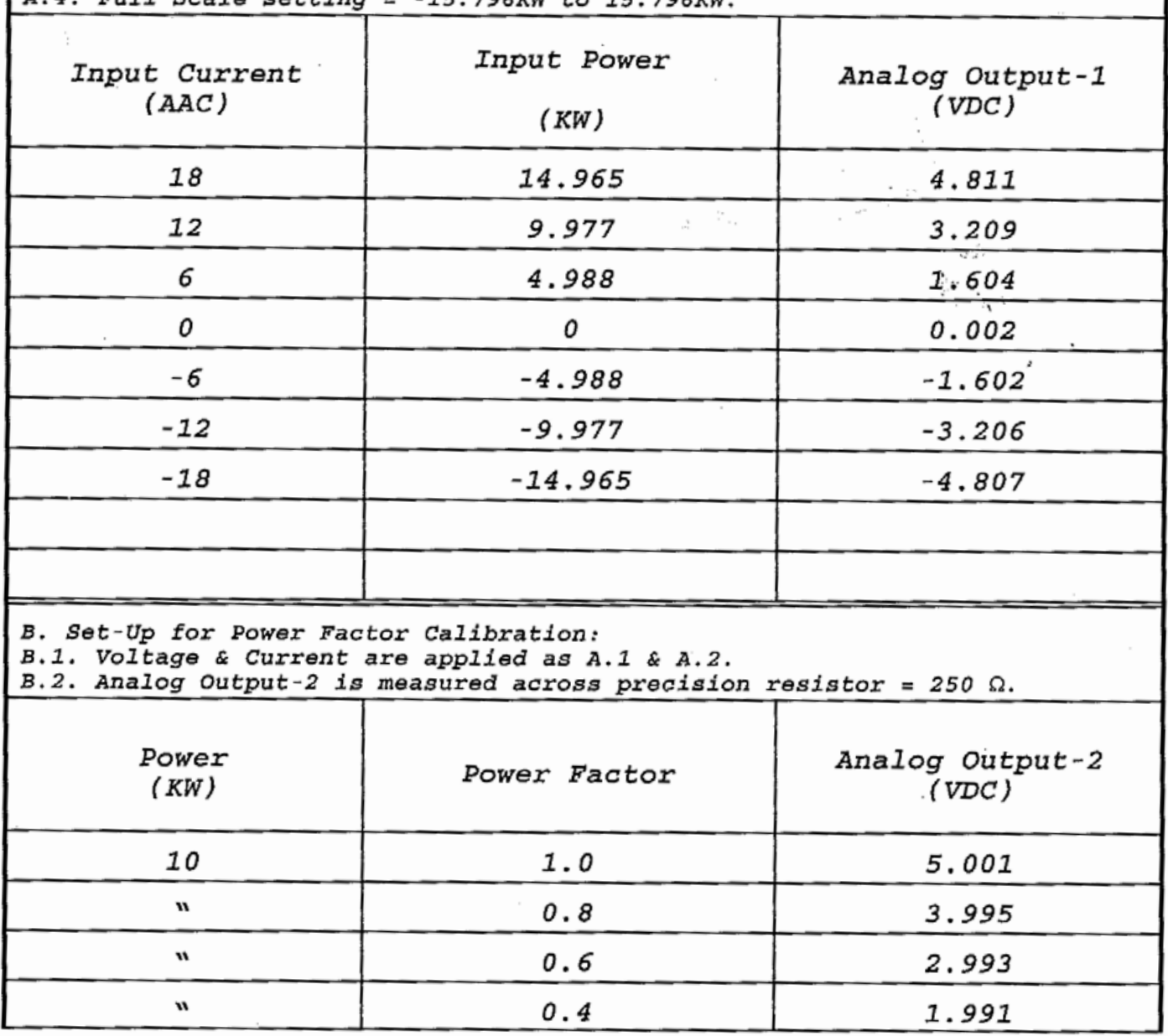

Page 1 of 2

Figure B.1. Power transducer calibration sheet 


\section{DEUTSCHER KALIBRIERDIENST DKD}

Kalibrierlaboratorium für Strömungsgeschwindigkeit von Luft Calibration laboratory for velocity of air flow

Akkreditiert durch die / accredited by the

Akkreditierungsstelle des DKD bei der PHYSIKALISCH-TECHNISCHEN BUNDESANSTALT (PTB)

\section{Deutsche WindGuard Wind Tunnel Services $\mathrm{GmbH}$ Varel}

\section{Kalibrierschein Calibration Certificate}

\begin{tabular}{|c|c|}
\hline $\begin{array}{l}\text { Gegenstand } \\
\text { Object }\end{array}$ & Cup Anemometer \\
\hline $\begin{array}{l}\text { Hersteller } \\
\text { Manufacturer }\end{array}$ & $\begin{array}{l}\text { Thies Clima } \\
\text { D-37083 Göttingen }\end{array}$ \\
\hline Typ & 4.3350 .00 .000 \\
\hline $\begin{array}{l}\text { Fabrikat/Serien-Nr. } \\
\text { Senial number }\end{array}$ & $\begin{array}{l}\text { Body: } 0707890 \\
\text { Cup: } 0707890\end{array}$ \\
\hline $\begin{array}{l}\text { Auftraggeber } \\
\text { Customer }\end{array}$ & $\begin{array}{l}\text { Thies Clima } \\
\text { D-37083 Göttingen }\end{array}$ \\
\hline $\begin{array}{l}\text { Auftragsnummer } \\
\text { Order No. }\end{array}$ & VT07255 \\
\hline \multicolumn{2}{|c|}{$\begin{array}{l}\text { Anzahl der Seiten des Kalibrierscheines } \\
\text { Number of pages of the certificate }\end{array}$} \\
\hline $\begin{array}{l}\text { Datum der Kalibrierung } \\
\text { Date of calibration }\end{array}$ & 24.07 .2007 \\
\hline
\end{tabular}

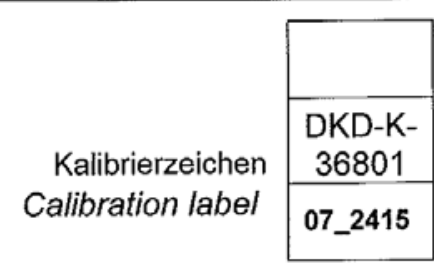

Dieser Kalibrierschein dokumentiert die Rückführung auf nationale Normale zur Darstellung der Einheiten in Öbereinstimmung mit dem Internationalen Einheitensystem (SI). Der DKD ist Unterzeichner der multi- lateralen Übereinkommen der European co-operation for Accreditation (EA) und der International Laboratory Accreditation Cooperation (ILAC) zur gegenseitigen Anerkennung der Kalibrierscheine.

Für die Einhaltung einer angemessenen Frist zur Wiederholung der Kalibrierung ist der Benutzer verantwortlich.

This calibration certificate documents the traceability to national standards, which realize the units of measurement according to the Intemational System of Units (SI).

The $D K D$ is signatory to the multilateral agreements of the European co-operation for Accreditation (EA) and of the Intemational Laboratory Accreditation Cooperation (ILAC) for the mutual recognition of calibration certificates.

The user is obliged to have the object recalibrated at appropriate intervals.

Dieser Kalibrierschein darf nur vollständig und unverändert weiterverbreitet werden. Auszüge oder Änderungen bedürfen der Genehmigung sowohl der Akkreditierungsstelle des DKD als auch des ausstellenden Kalibrierlaboratoriums. Kalibrierscheine ohne Unterschrift und Stempel haben keine Gültigkeit.

This calibration certificate may not be reproduced other than in full except with the permission of both the Accreditation Body of the DKD and the issuing laboratory. Calibration certificates without signature and seal are not valid.

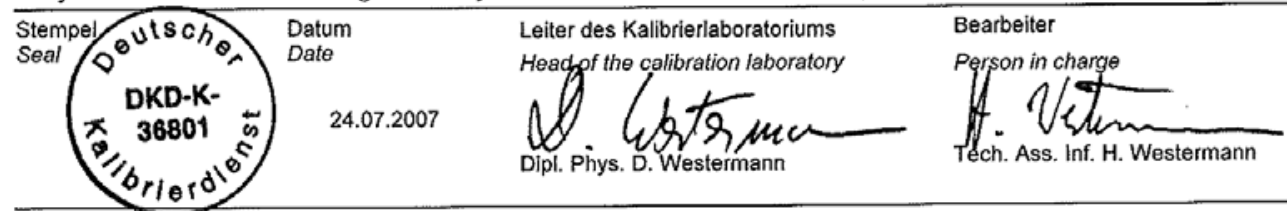

Deutsche WindGuard Wind Tunnel Services GmbH

Oldenburger Str. 65

26316 Varel ; Tel. ++49 (0)445195150

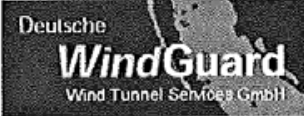

Figure B.2. Primary anemometer calibration sheet 


\section{DEUTSCHER KALIBRIERDIENST DKD}

Kalibrierlaboratorium für Strömungsgeschwindigkeit von Luft Calibration laboratory for velocity of air flow

Akkreditiert durch die / accredited by the

Akkreditierungsstelle des Deutschen Kalibrierdienstes

DEWIM

\section{DEWI GmbH \\ Deutsches Windenergie-Institut}

Kalibrierschein Calibration certificate

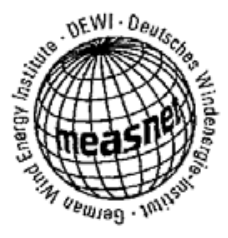

Cup Anemometer

Gegenstand

Object

Hersteller

Manufacturer

Typ

Type

Fabrikat/Serien-Nr. Serial number

Auftraggeber

Customer

Auftragsnummer

Order No.

Anzahl der Seiten des Kalibrierscheines

Number of pages of the certificate

Datum der Kalibrierung

Date of calibration

\section{Thies Clima \\ D-37083 Göttingen}

4.3350 .00 .000

body: 0707890

cup: -

Thies Clima

D-37083 Goettingen,

AB0901617

$3+3$

16.06.09
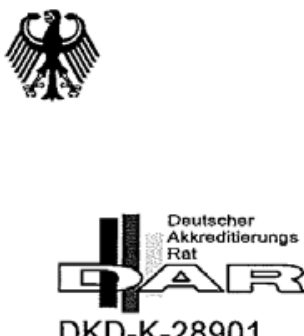

DKD-K-28901

Dieser Kalibrierschein darf nur vollständig und unverändert weiterverbreitet werden. Auszüge oder Änderungen bedurfen der Genehmigung sowohl der Akkreditierungsstelle des DKD als auch des ausstellenden Kalibrierlaboratoriums. Kalibrierscheine ohne Unterschrift und Stempel haben keine Gültigkeit.

This calibration certificate may not be reproduced other than in full except with the permission of both the Accreditation Body of the QKD and the issuing laboratory. Calibration certificates without signature and seal are not valid.

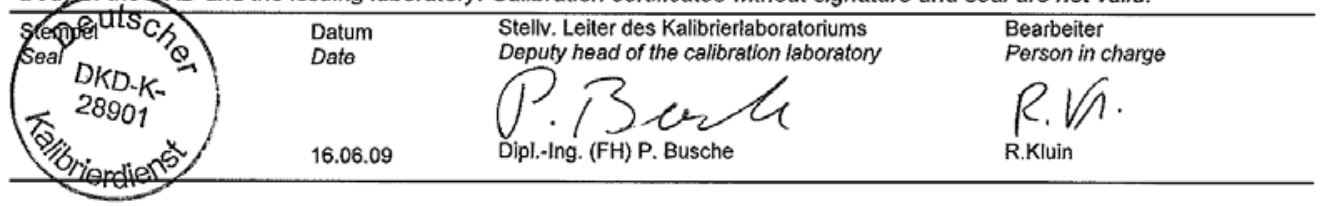

DEWI GmbH DEUTSCHES WINDENERGIE - INSTITUT Ebertstr. 96, D-26382 Wilhelmshaven

Tel. +49 (0)4421 4808-0, Fax. +49 (0)4421 4808-43

Figure B.3. Primary anemometer calibration sheet II 


\section{Wind Vane Calibration Report}
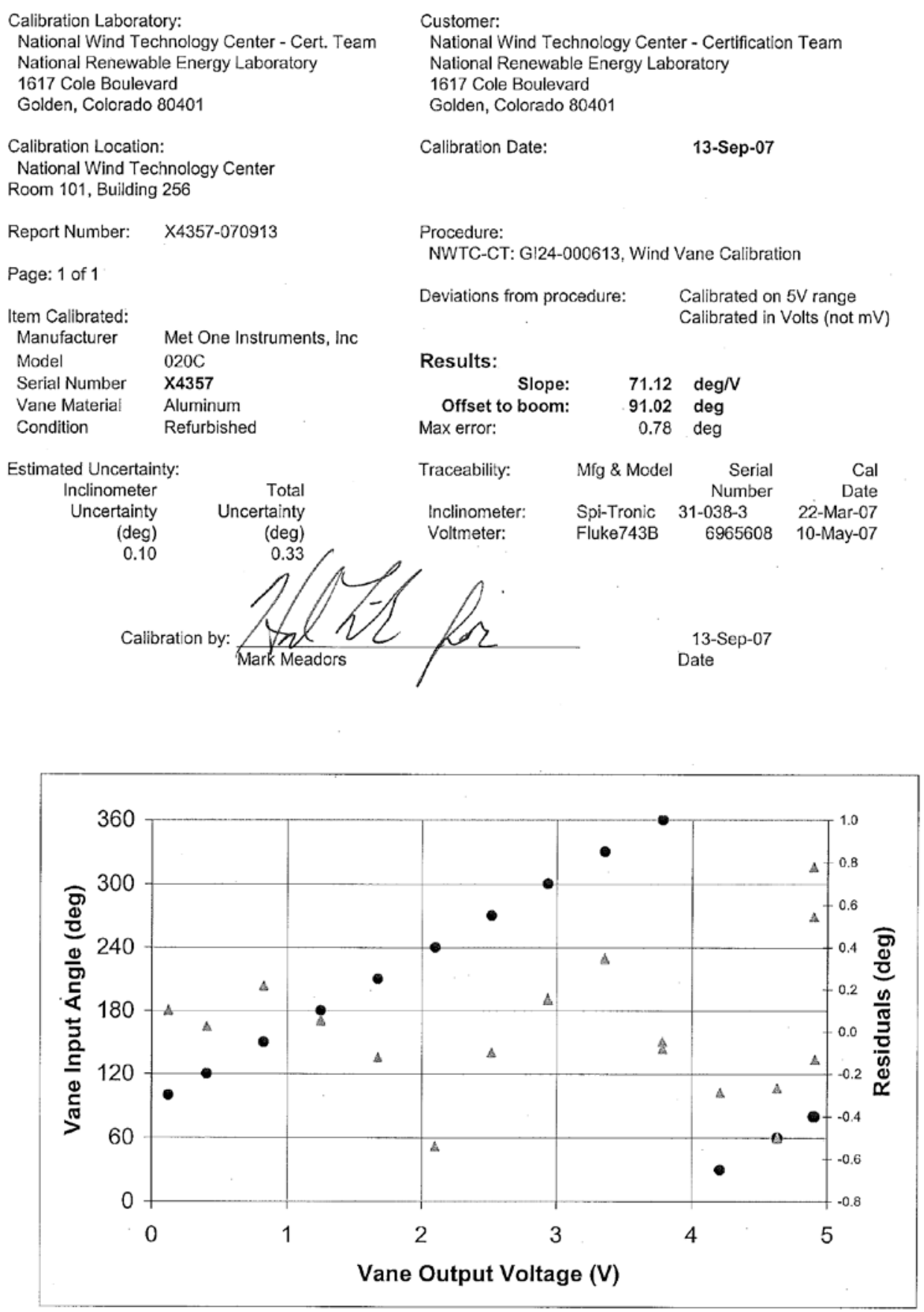

Figure B.4. Wind vane calibration sheet 


\section{NREL METROLOGY LABORATORY}

Test Report

Test Instrument: RTD Probe

DOE \#: $02885 C$

Model \# : 78NOINOON

$S / N \quad: 0890084$

Calibration Date: 10/29/2007

Due Date: 10/29/2008

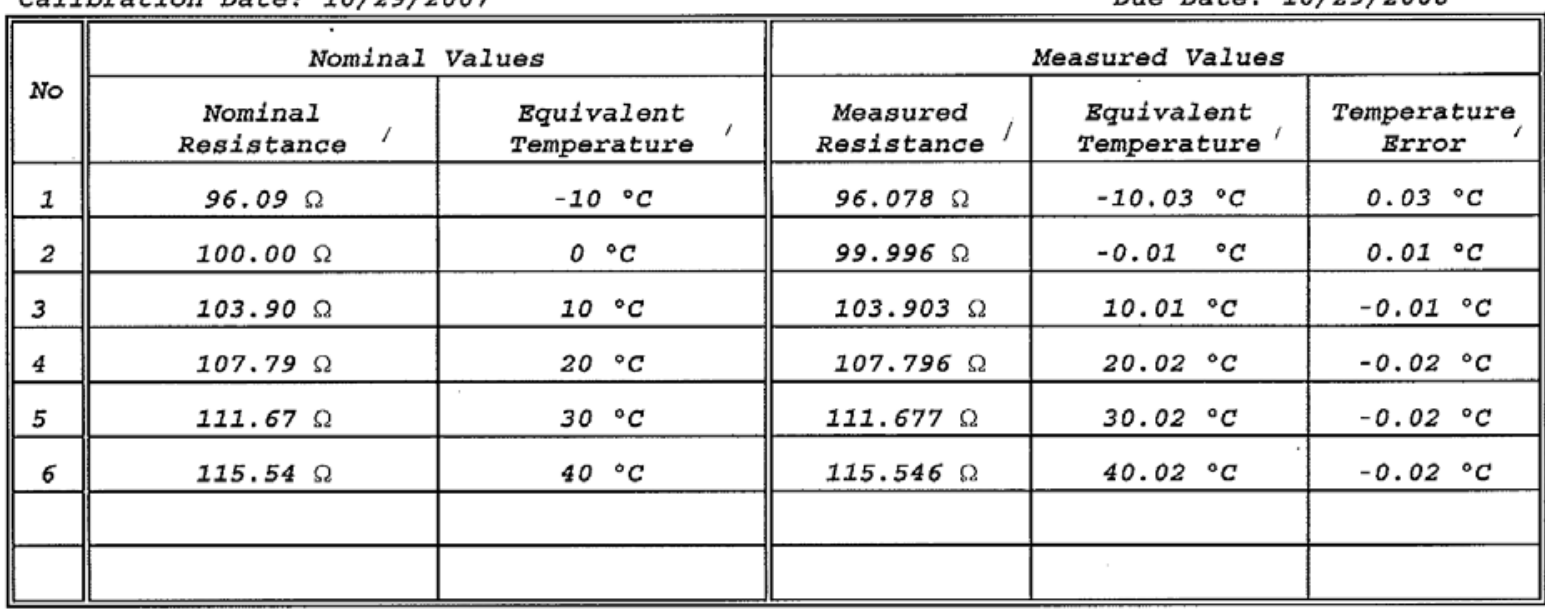

Notes:

1. Total Uncertainty of Nominal Values $= \pm 0.02{ }^{\circ} \mathrm{C}$

2. Calibration was performed at $23^{\circ} \mathrm{C}$ and $37^{\circ} \mathrm{RH}$

3. Resistance is measured using 4-wire technique 
NREL METROLOGY LABORATORY

Test Report

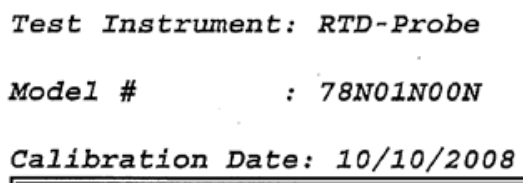

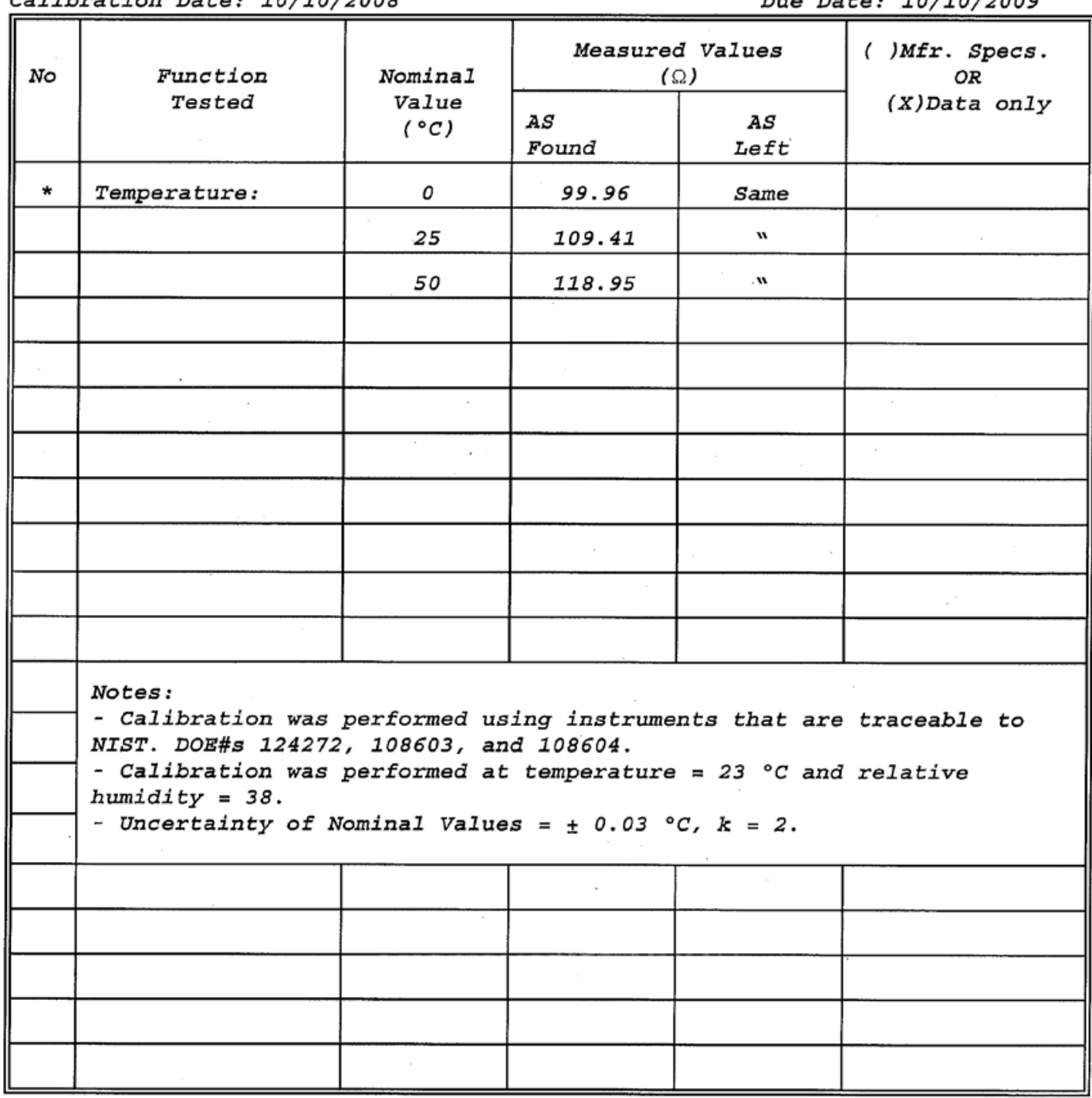

Tested By: Reda

Date

: $10 / 10 / 2008$

Figure B.6. RTD probe calibration sheet II 


\section{NREL METROLOGY LABORATORY}

\section{Test Report}

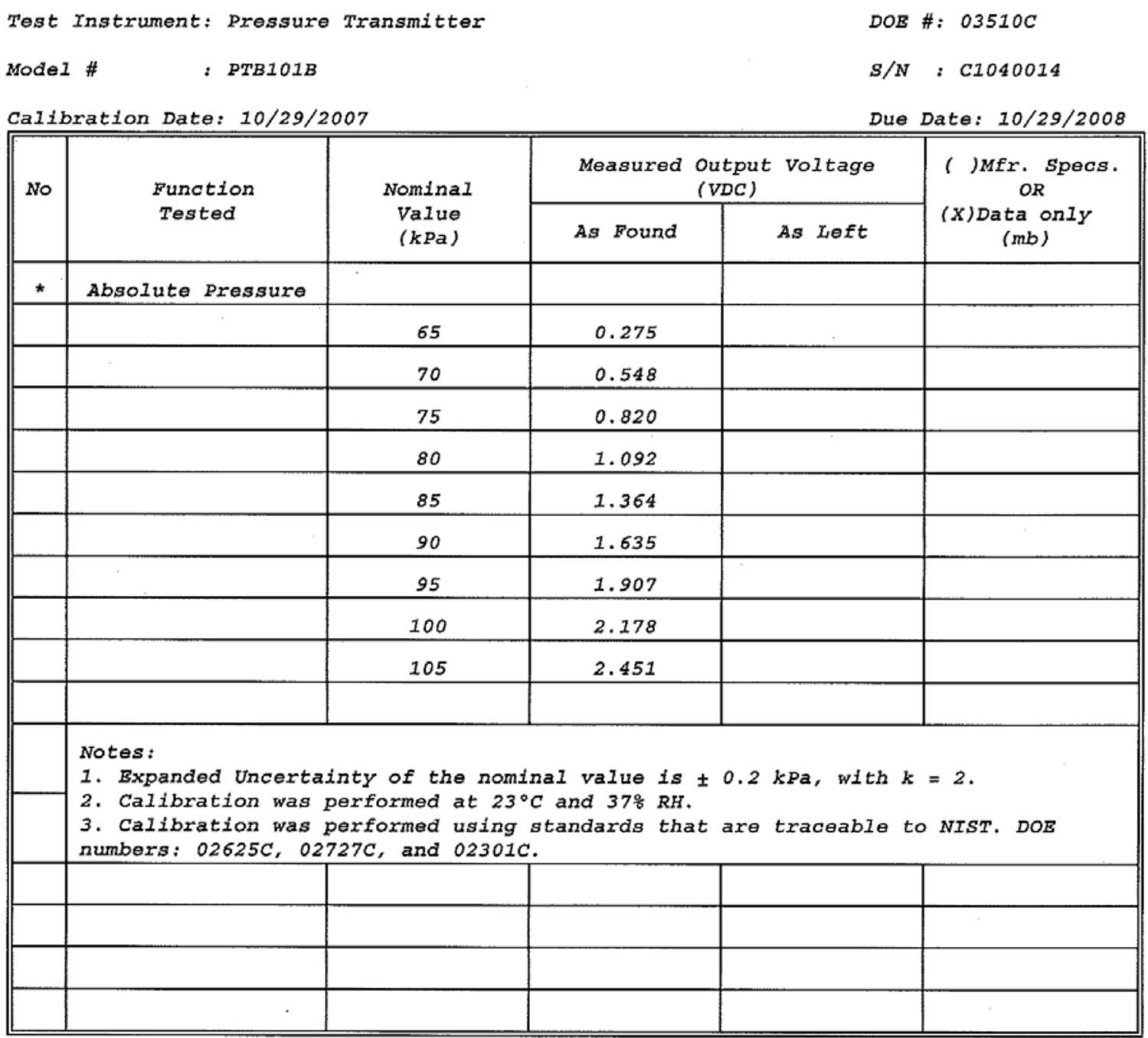

Figure B.7. Pressure transmitter calibration sheet 
Board Information:

Serial Number: 12CBC7A

NI Part Number: 192580D-02

Description: NI 9229

Calibration Date: 14-AUG-07

Recommended Calibration Due Date: 14-AUG-08*

Ambient Temperature: $23^{\circ} \mathrm{C}$

Relative Humidity: $60 \%$
Certificate Information:

Certificate Number: 793243

Date Printed: 20-NOV-08

National Instruments certifies that at the time of manufacture, the above product was calibrated in accordance with applicable National Instruments procedures. These procedures are in compliance with relevant clauses of ISO 9001 and are designed to assure that the product listed above meets or exceeds National Instruments specifications.

National Instruments further certifies that the measurements standards and instruments used during the calibration of this product are traceable to National and/or International Standards administered by NIST or Euromet members or are derived from accepted values of natural physical constants.

The environment in which this product was calibrated is maintained within the operating specifications of the instrument and the standards.

The information shown on this certificate applies only to the instrument identified above and the certificate may not be reproduced, except in full, without prior written consent by National Instruments.

For questions or comments, please contact National Instruments Technical Support.

NI Hungary Software és

Hardware Gyártó Kft.

4031 Debrecen, Határ út

$1 / A$.

HUNGARY
Signed,

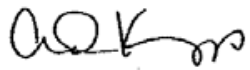

Andrew Krupp

Quality Director

* Recommended calibration due date is based on a combination of calibration interval and, when applicable, calibration shelf life. This date may vary depending on your application requirements.

Figure B.8. NI 9229 data acquisition module calibration sheet I 
Board Information:

Serial Number: 12BFEE2

NI Part Number: 192547D-01

Description: NI 9217

Calibration Date: 20-JUL-07

Recommended Calibration Due Date: 20-JUL-08*

Ambient Temperature: $26^{\circ} \mathrm{C}$

Relative Humidity: $45 \%$
Certificate Information:

Certificate Number: 775348

Date Printed: 20-NOV-08

National Instruments certifies that at the time of manufacture, the above product was calibrated in accordance with applicable National Instruments procedures. These procedures are in compliance with relevant clauses of ISO 9001 and are designed to assure that the product listed above meets or exceeds National Instruments specifications.

National Instruments further certifies that the measurements standards and instruments used during the calibration of this product are traceable to National and/or International Standards administered by NIST or Euromet members or are derived from accepted values of natural physical constants.

The environment in which this product was calibrated is maintained within the operating specifications of the instrument and the standards.

The information shown on this certificate applies only to the instrument identified above and the certificate may not be reproduced, except in full, without prior written consent by National Instruments.

For questions or comments, please contact National Instruments Technical Support.

NI Hungary Software és

Hardware Gyártó Kft.

4031 Debrecen, Határ út

$1 / A$.

HUNGARY
Signed,

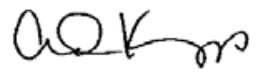

Andrew Krupp

Quality Director

* Recommended calibration due date is based on a combination of calibration interval and, when applicable, calibration shelf life. This date may vary depending on your application requirements.

Figure B.9. NI 9217 data acquisition module calibration sheet I 
Board Information:

Serial Number: 12E9C99

NI Part Number: 193299F-01

Description: NI-9205

Calibration Date: 08-OCT-07

Recommended Calibration Due Date: 08-OCT-08*

Ambient Temperature: $23^{\circ} \mathrm{C}$

Relative Humidity: $38 \%$

\section{Certificate Information:}

Certificate Number: 835019

Date Printed: 20-NOV-08

National Instruments certifies that at the time of manufacture, the above product was calibrated in accordance with applicable National Instruments procedures. These procedures are in compliance with relevant clauses of ISO 9001 and are designed to assure that the product listed above meets or exceeds National Instruments specifications.

National Instruments further certifies that the measurements standards and instruments used during the calibration of this product are traceable to National and/or International Standards administered by NIST or Euromet members or are derived from accepted values of natural physical constants.

The environment in which this product was calibrated is maintained within the operating specifications of the instrument and the standards.

The information shown on this certificate applies only to the instrument identified above and the certificate may not be reproduced, except in full, without prior written consent by National Instruments.

For questions or comments, please contact National Instruments Technical Support.

NI Hungary Software és

Hardware Gyártó Kft.

4031 Debrecen, Határ út

$1 / A$.

HUNGARY
Signed,

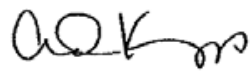

Andrew Krupp Quality Director

* Recommended calibration due date is based on a combination of calibration interval and, when applicable, calibration shelf life. This date may vary depending on your application requirements.

Figure B.10. NI 9205 data acquisition module calibration sheet I 


\section{Davis|Calibration}

Company ID: 229037

NATIONAL INSTRUMENTS

11500 N. MOPAC EXPWY

ATTN. RMA DEPT.

AUSTIN, TX78759

Instrument ID: 12CBC7A

Manufacturer. NATIONAL INSTRUMENTS

Description: 4-CHANNEL, $\pm 60 \mathrm{~V}, 24-\mathrm{BIT}$ SIMULTANEOUS ANALOG INPUT

Instrument Identification

\section{Certificate of Calibration}

3214337

Certificate Page 1 of 1

PO Number: 337683

Model Number: N1 9229

Serial Number: $12 \mathrm{CBC} 7 \mathrm{~A}$

Accuracy: Mr Specifications

Certificate Information

Reason For Service: CALIBRATION

Type of Cal: ACCREDITED 17025

As Found Condition: IN TOLERANCE

As Left Condition: LEFT AS FOUND

Procedure: NATIONAL INSTRUMENTS CAL EXECUTIVE REV 3.3.1

Remarks: Reference attached Data.
Technician: WAYNE GETCHELL Cal Date 06May2009

Cal Due Date: $06 \mathrm{May} 2010$ Interval: 12 MONTHS

Temperature: $23.0 \mathrm{C}$ Humidity: $44.0 \%$

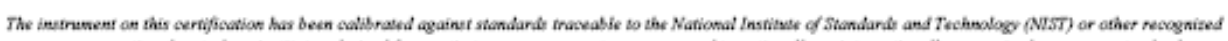

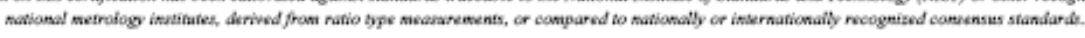

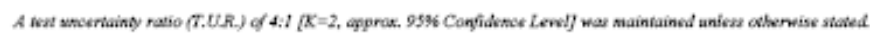

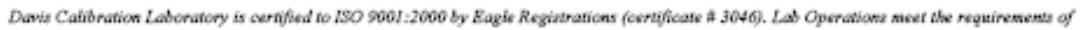

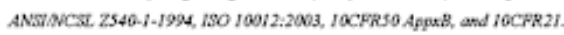

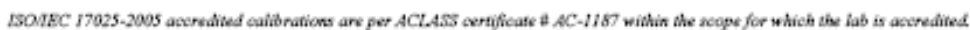

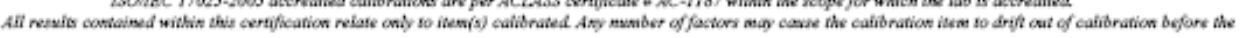
instrament's cefichation intervat hat expired.

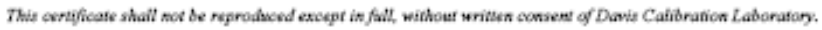

Approved By: VICTOR PENA

Service Representative

\begin{tabular}{|c|c|c|c|c|c|}
\hline \multicolumn{6}{|c|}{ Calibration Standards } \\
\hline NIST Traceable\# & Inst. ID\# & Description & Model & Cal Date & Date Due \\
\hline 3143038 & $15-0271$ & MULTIFUNCTION CALBBRATOR & $5700 \mathrm{~A}$ & 15Apr2009 & 14Jul2008 \\
\hline
\end{tabular}

Davis Calibration • 2324 Ridgepoint Drive, Suite D • Austin, TX 78754 • Phone: 800-365-0147 • Fax: 512-926-8450

Figure B.11. NI 9229 data acquisition module calibration sheet II 


\section{Davis Calibration}

Company ID: 229037

NATIONAL INSTRUMENTS

11500 N. MOPAC EXPWY

ATTN. RMA DEPT.

AUSTIN, TX 78759

Instrument ID: 12BFEE2

Manufacturer: NATIONAL INSTRUMENTS

Description: 4-CH 100 OHM 24-BIT RTD ANALOG INPUT

Accuracy: Mfr. Specifications

\section{Certificate of Calibration}

3214181

Certificate Page 1 of 1

Instrument Identification

PO Number: 337683

Model Number: NI 9217

Serial Number: 12BFEE2

\section{Certificate Information}

Reason For Service: CALIBRATION

Type of Cal: ACCREDITED 17025

As Found Condition: IN TOLERANCE

As Left Condition: LEFT AS FOUND

Procedure: CAL EXEC 3.3.1 CAL EXEC 3.3.1

Remarks: Reference attached Data.
Technician: WAYNE GETCHELL Cal Date 06May2009

Cal Due Date: 06May 2010 Interval: 12 MONTHS

Temperature: $23.0 \mathrm{C}$ Humidity. $46.0 \%$

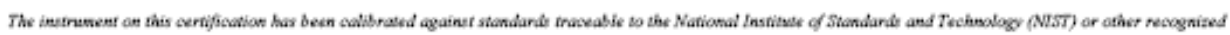

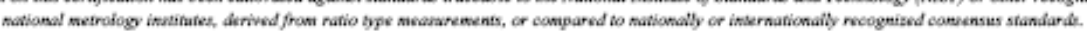

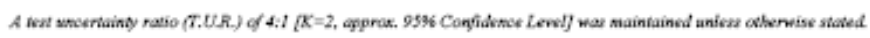

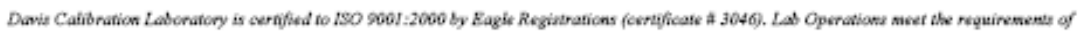

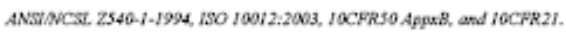

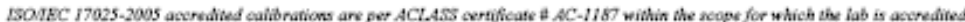

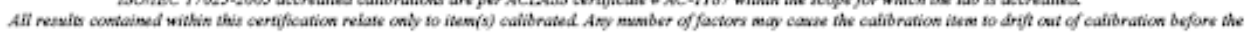
instrawent's calit ration istervat hat expired.

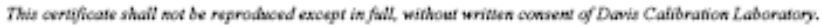

Approved By: VICTOR PENA

Service Representative

Calibration Standards

\begin{tabular}{|c|c|c|c|c|c|}
\hline NIST Traceable\# & Inst. ID\# & Description & Model & Cal Date & Date Due \\
\hline 3078982 & $15-0011$ & DECADERESSTOR & $\mathrm{DB} 52$ & $24 \operatorname{Mar} 2000$ & $24 \mathrm{Mar} 2010$ \\
\hline 3004176 & $15-0060$ & DIGTAL MULTIMETER (GOLDEN CAL) & 345BA OPT 002 & 17Feb2009 & 17May200e \\
\hline
\end{tabular}

Davis Calibration • 2324 Ridgepoint Drive, Suite D • Austin, TX 78754 • Phone: 800-365-0147 • Fax: 512-926-8450

Figure B.12. NI 9217 data acquisition module calibration sheet II 


\section{Davis Calibration}

Company ID: 229037

NATIONAL INSTRUMENTS

11500 N. MOPAC EXPWY

ATTN. RMA DEPT.

AUSTIN, TX 78759

Instrument ID: 12E9C99

Manufacturer: NATIONAL INSTRUMENTS

Description: $32-\mathrm{CH} \pm 200 \mathrm{MV}$ TO $\pm 10 \mathrm{~V}, 16-\mathrm{BIT}, 250 \mathrm{KS} / \mathrm{S}$ ANALOG INPUT MODULE

Instrument Identification

PO Number: 337683

Model Number: NI 9205

Serial Number: 12E9C99

\section{Certificate of Calibration}

3214135

Certificate Page 1 of 1

Accuracy: Mrr Specifications

Certificate Information

Technician: WAYNE GETCHELL Cal Date 06May2009 Cal Due Date: 06May2010 Interval: 12 MONTHS

Temperature: $23.0 \mathrm{C}$ Humidity. $47.0 \%$

Remarks: Reference attached Data.

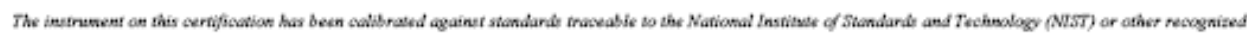

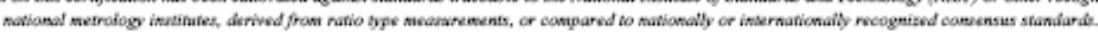

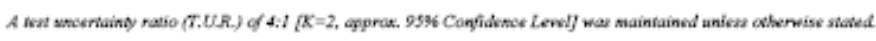

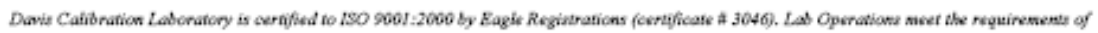

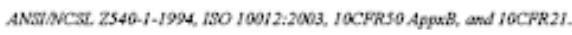

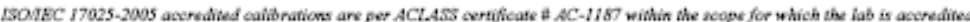

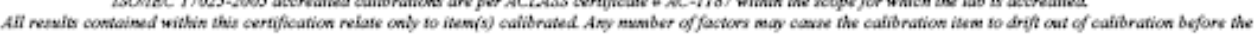
instrament's cation ration isterval hat expired.

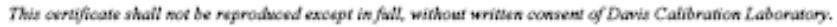

Approved By: VICTOR PENA

Service Representative

\begin{tabular}{|c|c|c|c|c|c|}
\hline \multicolumn{6}{|c|}{ Calibration Standards } \\
\hline NIST Traceable\# & Inst. ID\# & Description & Model & Cal Date & Date Due \\
\hline 3143038 & $15-0271$ & MULTIFUNCTION CALBRATOR & $5700 \mathrm{~A}$ & 15Apr2009 & 14Jul2008 \\
\hline
\end{tabular}

Davis Calibration • 2324 Ridgepoint Drive, Suite D • Austin, TX 78754 • Phone: 800-365-0147 • Fax: 512-926-8450

Figure B.13. NI 9205 data acquisition module calibration sheet II 


\section{REPORT DOCUMENTATION PAGE}

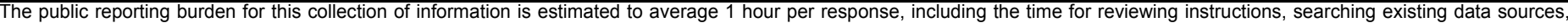

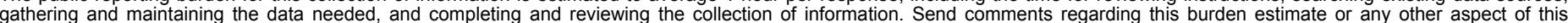

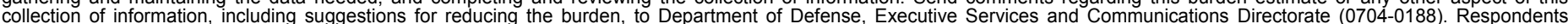

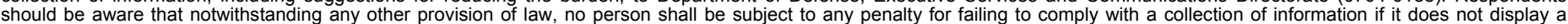

should be aware that notwithstanding

PLEASE DO NOT RETURN YOUR FORM TO THE ABOVE ORGANIZATION.

\begin{tabular}{l|l|l|l} 
1. REPORT DATE $(D D-M M-Y Y Y Y)$ & 2. & REPORT TYPE & 3. DATES COVERED (FrOm - TO)
\end{tabular}

December 2009

4. TITLE AND SUBTITLE

Wind Turbine Generator System Power Performance Test Report

for the Gaia-Wind 11-kW Wind Turbine

5a. CONTRACT NUMBER

DE-AC36-08-GO28308

5b. GRANT NUMBER

5c. PROGRAM ELEMENT NUMBER

6. AUTHOR(S)

A. Huskey, A. Bowen, and D. Jager

5d. PROJECT NUMBER

NREL/TP-500-46151

5e. TASK NUMBER

WER94501

5f. WORK UNIT NUMBER

7. PERFORMING ORGANIZATION NAME(S) AND ADDRESS(ES)

National Renewable Energy Laboratory

1617 Cole Blvd. REPORT NUMBER

Golden, CO 80401-3393

NREL/TP-500-46151

9. SPONSORING/MONITORING AGENCY NAME(S) AND ADDRESS(ES)

10. SPONSOR/MONITOR'S ACRONYM(S) NREL

11. SPONSORING/MONITORING AGENCY REPORT NUMBER

12. DISTRIBUTION AVAILABILITY STATEMENT

National Technical Information Service

U.S. Department of Commerce

5285 Port Royal Road

Springfield, VA 22161

13. SUPPLEMENTARY NOTES

14. ABSTRACT (Maximum 200 Words)

This test is being conducted as part of the U.S. Department of Energy's (DOE) Independent Testing project. It is a power performance test that the National Renewable Energy Laboratory (NREL) conducted on the Gaia-Wind 11-kW small wind turbine.

15. SUBJECT TERMS

Gaia wind turbine; power performance test; 11-kW; small wind turbine

\begin{tabular}{|c|c|c|}
\hline $\begin{array}{l}\text { a. REPORT } \\
\text { Unclassified }\end{array}$ & $\begin{array}{l}\text { b. ABSTRACT } \\
\text { Unclassified }\end{array}$ & $\begin{array}{l}\text { c. THIS PAGE } \\
\text { Unclassified }\end{array}$ \\
\hline
\end{tabular}

\begin{tabular}{l|l} 
17. LIMITATION & 18. $\begin{array}{l}\text { NUMBER } \\
\text { OF ABSTRACT } \\
\text { OF PAGES }\end{array}$ \\
UL &
\end{tabular}

19a. NAME OF RESPONSIBLE PERSON

19b. TELEPHONE NUMBER (Include area code) 